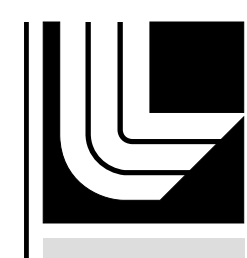

LAWRENCE LIVERMORE N A T IO N A L LABORATORY

Onset of Convection in Two

Liquid Layers with Phase Change

G. B. McFadden, S. R. Coriell, K. F. Gurski, D. L. Cotrell

September 20, 2006

Physics of Fluids 
This document was prepared as an account of work sponsored by an agency of the United States Government. Neither the United States Government nor the University of California nor any of their employees, makes any warranty, express or implied, or assumes any legal liability or responsibility for the accuracy, completeness, or usefulness of any information, apparatus, product, or process disclosed, or represents that its use would not infringe privately owned rights. Reference herein to any specific commercial product, process, or service by trade name, trademark, manufacturer, or otherwise, does not necessarily constitute or imply its endorsement, recommendation, or favoring by the United States Government or the University of California. The views and opinions of authors expressed herein do not necessarily state or reflect those of the United States Government or the University of California, and shall not be used for advertising or product endorsement purposes. 


\title{
Onset of Convection in Two Liquid Layers with Phase Change
}

\author{
By G. B. McFADDEN ${ }^{1}$, S. R. CORIELL $L^{1}$ K. F. GURSKI $\mathrm{I}^{2}$, \\ AND D. L. COTRELL $L^{3}$ \\ ${ }^{1}$ National Institute of Standards and Technology, Gaithersburg, MD 20899, USA \\ ${ }^{2}$ Department of Mathematics, George Washington University, Washington, DC 20052, USA \\ ${ }^{3}$ Lawrence Livermore National Laboratory, Livermore, CA 94550, USA
}

(Received ?? and in revised form ??)

We perform linear stability calculations for horizontal fluid bilayers that can undergo a phase transformation, taking into account both buoyancy effects and thermocapillary effects in the presence of a vertical temperature gradient. We compare the familiar case of the stability of two immiscible fluids in a bilayer geometry with the less-studied case that the two fluids represent different phases of a single-component material, e.g., the water-steam system. The two cases differ in their interfacial boundary conditions: the condition that the interface is a material surface is replaced by the continuity of mass flux across the interface, together with an assumption of thermodynamic equilibrium that in the linearized equations represents the Clausius-Clapeyron relation relating the interfacial temperature and pressures. For the two-phase case, we find that the entropy difference between the phases plays a crucial role in determining the stability of the system. For small values of the entropy difference between the phases, the two-phase system can be linearly unstable to either heating from above or below. The instability is due to the Marangoni effect in combination with the effects of buoyancy (for heating from below). For larger values of the entropy difference the two-phase system is unstable only for heating from below, and the the Marangoni effect is masked by effects of the entropy difference. To help understand the mechanisms driving the instability on heating from below we have performed both long-wavelength and short-wavelength analyses of the two-phase system. The short-wavelength analysis shows that the instability is driven by a coupling between the flow normal to the interface and the latent heat generation at the interface. The mechanism for the large wavelength instability is more complicated, and the detailed form of the expansion is found to depend on the Crispation and Bond numbers as well as the entropy difference. The two-phase system allows a conventional Rayleigh-Taylor instability if the heavier fluid overlies the lighter fluid; applying a temperature gradient allows a stabilization of the interface.

\section{Introduction}

The study of the stability of a fluid-fluid interface is important in a number of scientific and technological applications. In this paper we consider two fluid layers separated by a horizontal planar interface subject to a vertical temperature gradient. The case of two fluids that are, to a greater or lesser degree, immiscible has been well studied both theoretically and experimentally (Davis 1987; Joseph \& Renardy 1993; Johnson \& Narayanan 1998; Andereck et al. 1998; Schatz \& Neitzel 2001; Nepomnyashchy et al. 2002), and the 
effects of various driving forces on the stability of the system have been taken into account. Examples include the effects of buoyancy [natural or Rayleigh-Benard convection (Turner 1973)], the effects of bulk density differences [Rayleigh-Taylor instabilities (Chen $\&$ Fried 2006)], and the effects of surface tension gradients along the interface [Marangoni instabilities (Davis 1987)].

This situation can be contrasted with that of a bilayer system in which the two layers represent different phases of a single fluid (Busse \& Schubert 1971; Busse 1989; Sakurai et al. 1999). The phase transformation that may then occur between the two layers is described by a modification of the usual interfacial boundary conditions that are used to describe immiscible fluids. For a two-phase system there can be mass flow across the interface, which is not a material surface. In addition, latent heat is typically generated at the interface which is conducted into the surrounding fluid. Finally, a description of the thermodynamic state of the interface is required, which is often based either on an assumption of local thermodynamic equilibrium or a kinetic statement governing systematic deviations from local thermodynamic equilibrium. As a result, the stability results for a two-phase bilayer system are quantitatively and even qualitatively different than that of an immiscible system. To simplify our terminology, we shall refer to a system consisting of two immiscible fluids as the "inert case," and shall refer to a bilayer consisting of different phases of a single fluid as the "two-phase case." Examples of the two-phase case arise in many material processing applications.

Many studies of bilayer instabilities of the inert case consider two fluids with materials properties that are sufficiently different in the two phases that one of the layers is passive, and can be neglected to produce a simpler single layer system. In addition, if the effects of surface tension are strong enough the deflection of the interface may be neglected, resulting in further simplification of the analysis. In contrast, two-phase systems often arise near the thermodynamic critical point of a single component fluid or in cases of phase-separation in multicomponent systems. In these systems, the material constants in each phase are often of similar magnitude, particularly close to the transition points where the two phases become indistinguishable, and the simplifications employed for the inert case may not apply for the two-phase case. Examples of single component systems include the water-steam system as used in the power industry, and examples of phaseseparating multicomponent systems include the cases of spinodal decomposition and of monotectic growth.

This paper was initially motivated by the question of wavelength selection in the directional processing of monotectic systems. In these systems a single liquid phase (the "parent" phase) transforms into two "daughter" phases, which consist of a solid phase and another liquid phase with properties that are distict from the parent phase (Hunt \& $\mathrm{Lu}$ 1994). The daughter phases often take the form of an array of liquid rod inclusions in a solid matrix. Spacing predictions for the geometry that ignore the effects of convection produce results that are in significant disagreement with experimental observations (Andrews et al. 2006), suggesting the consideration of models that include flow effects such as Rayleigh-Benard, Rayleigh-Taylor, and Marangoni convection (Stöcker \& Ratke 2000). Fluid flow due to thermocapillary effects is suspected to play an especially important role during growth under microgravity conditions.

To estimate the relative importance of these types of instabilities for a two-phase system, we consider a simplified bilayer geometry in which a horizontal interface separates two semi-infinite layers of a single-component fluid. This problem is still sufficiently complex that we generally resort to a numerical determination of the linear stability of the system, including the possibility of oscillatory modes and significant interfacial deformations. The governing equations contain a large number of dimensionless parameters, 
including a Rayleigh number, Marangoni number, Bond number, Crispation number, and ratios of material properties of the two phases. To validate the numerical procedure, we compare with the previous treatment of the inert case by Zeren \& Reynolds (1972) for a benzene-water system [see also Ferm \& Wollkind (1982)]. We then consider a second case with material parameters appropriate for the water-steam system. For comparison purposes, the two-phase results for the water-steam system are contrasted with those for a hypothetical inert system with similar material properties. For the two-phase case, we find particularly interesting stability results at low wavenumbers, for a mode that is sensitive to the differences in enthalpy and entropy between the two phases. To examine this mode in more detail we perform a small-wavenumber expansion of the stability problem, finding that the mode is also sensitive to the values of both the Bond and Crispation numbers of the system. A similar small-wavenumber analysis was performed by Zeren \& Reynolds (1972) for the inert case, which we reproduce (with minor corrections) for comparison purposes.

For simplicity we assume that local equilibrium holds at the fluid-fluid interface. More general boundary conditions that apply under non-equilibrium conditions have been discussed by a number of authors, including Anderson et al. (2006). These conditions can also be derived by considering sharp-interface limits (Anderson et al. 2001) of diffuse interface models (Edwards et al. 1991; Anderson et al. 1998, 2000).

We find that a two-phase system that is heated from below is subject to a type of morphological instability at small wavenumbers that is similar in origin to other, more familiar, instabilities that occur in materials processing applications (Davis 2001). Multiphase systems are typically subject to instability if one or more of the phases is in a thermodynamic state of metastability; specifically, if there are regions of space where the actual phase of the system is not the phase of lowest free energy under the given conditions. Examples include superheating a solid above its melting point, supercooling a liquid below its melting point, supersaturating a solution beyond its solubility limit, or compressing a gaseous component beyond its vapour pressure. For growth of a single component crystal from its supercooled liquid phase the instability can lead to dendritic structures (Langer 1980; Huang \& Glicksman 1981); a stability analysis was performed by Mullins \& Sekerka (1963). A crystal of binary alloy growing from its melt is subject to instability if the liquid ahead of the interface is "constitutionally supercooled" (Tiller et al. 1953); a stability analysis of this situation was also performed by Mullins \& Sekerka (1964). In both cases, the driving force for an instability is the release of free energy accompanying a phase transformation from the higher energy phase to the lower energy phase. In practice the nucleation of the lower energy phase in the bulk unstable phase is rarely observed under conditions of mild disequilibrium, since there is an accompanying energy penalty associated with the creation of surface area of the new phase. However the energy difference can drive the instability at an interface where the two phases come in contact. The resulting instability generally displays a wavelength that is determined by a balance between surface energy (stabilizing) and the difference in bulk free energy between the phases (destabilizing).

A portion of the phase diagram for a representative water-steam system is depicted in Fig. 1. A two-phase system consisting of liquid and gas is in thermodynamic equilibrium along the co-existence curve in the pressure-temperature state space. The coexistence curve terminates at the thermodynamic critical point where the properties of the liquid and gas become equal. The liquid phase is preferred under conditions of higher pressure and lower temperature, and the gas phase is preferred for lower pressures and higher temperatures. The slope of the co-existence curve is given by the Clausius-Clapeyron 
equation (Callen 1960),

$$
\frac{d p}{d T}=\frac{\left(s_{m}^{\alpha}-s_{m}^{\beta}\right)}{\left(1 / \bar{\rho}_{\alpha}-1 / \bar{\rho}_{\beta}\right)},
$$

where $s_{m}^{\alpha}$ and $s_{m}^{\beta}$ are the entropy densities of the phases, and $\bar{\rho}_{\alpha}$ and $\bar{\rho}_{\beta}$ are the densities; here we assume the lighter gas phase $(\alpha)$ overlies the denser liquid phase $(\beta)$. If the bilayer is heated from below, the static pressure and temperature profiles $p(z)$ and $T(z)$ in each phase satisfy $d p=-\rho g d z$ and $d T=G d z$, where $z$ is the vertical coordinate, $g$ is the gravitational acceleration and $G$ denotes the temperature gradient, whch is negative for heating from below. The condition that the liquid is superheated or that the gas is supercooled relative to thermodynamic equilibrium can be determined graphically by plotting the pressure and temperature profiles $p(z)$ and $T(z)$ in relation to the co-existence curve, or by comparing the slopes $d p / d T=-\rho g / G$ at the interface in each phase with that given by the Clausius-Clapeyron equation. This relation gives the correct qualitative form for the observed long-wave instability, but is not quantitively accurate since the instability involves lateral pressure and temperature gradients along the interface that drive convection and alter the thermal transport in the system. Our results include the derivation of a quantitative version of this expression that is obtained by performing a small-wavenumber expansion for the full governing equations, including the effects of convection. The predictions are in good agreement with the corresponding numerical results that we obtain.

The paper is organized as follows. Governing equations for the inert and two-phase cases are given in the next section, including a discussion of the different interfacial conditions for the inert and two-phase cases. The numerical procedure is described briefly in Section 3. Linear stability results for various cases are presented next, including a comparison of the numerical results with large and small wavenumber expansions. A discussion is presented in Section 5, followed by conclusions in Section 6. An appendix contains a summary of the expansion results.

\section{Equations}

We consider a semi-infinite horizontal two layer system, with vertical heating across the layers. The unperturbed upper layer (denoted by $\alpha$ ) extends over the interval $0<z<H_{\alpha}$, and the unperturbed lower layer (denoted by $\beta$ ) extends over the interval $-H_{\beta}<z<$

0 . Without loss of generality we consider linear stability results for a two-dimensional system. The horizontal coordinate extends from $-\infty<x<\infty$, and the velocity $\mathbf{u}$ has components in the $x$ and $z$ directions given by $u$ and $w$, respectively.

\subsection{Governing Equations in the Bulk}

In each phase, we consider the Boussinesq equations

$$
\begin{gathered}
\nabla \cdot \mathbf{u}=0, \\
\bar{\rho} u_{t}+\bar{\rho}(\mathbf{u} \cdot \nabla) \mathbf{u}+\nabla p=\mu \nabla^{2} \mathbf{u}-\rho g \mathbf{z}, \\
T_{t}+(\mathbf{u} \cdot \nabla) T=\kappa \nabla^{2} T,
\end{gathered}
$$

Here $p$ is the pressure, $\mu$ is the dynamic viscosity, $T$ is the temperature, $\kappa$ is the thermal diffusivity, $g$ is the gravitational acceleration, $t$ is the time, and $\mathbf{z}$ is the unit vector in the $z$-direction (anti-parallel to gravity). We assume $\mu$ and $\kappa$ are uniform in each phase, and also assume the density $\rho$ is uniform in all terms except the gravitational term, where 
the density is given by

$$
\rho=\bar{\rho}\left(1-\eta\left[T-T_{R}\right]\right) .
$$

Here $\bar{\rho}$ is the density in each phase at the reference temperature $T_{R}$, and the thermal expansion coefficient $\eta$ is assumed to be uniform in each phase.

\subsection{Boundary Conditions}

The upper boundary at $z=H_{\alpha}$ and the lower boundary at $z=-H_{\beta}$ are assumed to be isothermal with no-slip boundary conditions. We will consider two sets of boundary conditions at the interface between the two fluids: one set is appropriate to two immiscible liquids ("inert system"), and the other for a phase boundary for a single-component fluid system ("two-phase system"). The interface is assumed to have the form $z=h(x, t)$.

Both systems have a common subset of boundary conditions. The temperature is continuous across the interface,

$$
\llbracket T \rrbracket=0,
$$

where $\llbracket T \rrbracket=T^{\alpha}-T^{\beta}$ denotes the temperature jump across the interface. The tangential velocity is assumed to satisfy the no-slip condition

$$
\llbracket \mathbf{u} \cdot \mathbf{t} \rrbracket=0,
$$

where $\mathbf{t}$ is any tangent vector to the interface. The stress boundary condition is

$$
\llbracket \rho \mathbf{u}\left(\mathbf{u} \cdot \mathbf{n}-v_{n}\right) \rrbracket=\llbracket \mathbb{T} \cdot \mathbf{n} \rrbracket-\gamma \mathcal{K} \mathbf{n}+\nabla_{S} \gamma,
$$

where $\mathbf{n}$ is a unit normal vector to the interface, $\mathbb{T}_{j k}=-p \delta_{j k}+\mu\left(\partial u_{j} / \partial x_{k}+\partial u_{k} / \partial x_{j}\right)$ is the stress tensor, $\gamma$ is the surface tension, $\mathcal{K}$ is (twice) the mean curvature, $v_{n}$ is the normal velocity of the interface, and $\nabla_{S}$ is the surface gradient. Here our sign convention is that the curvature $\mathcal{K}$ is defined to be positive for a spherical inclusion of $\beta$ phase. For example, in two dimensions with an interface $y=h(x, t)$, the curvature is $\mathcal{K}=-h_{x x} /\left[1+h_{x}^{2}\right]^{3 / 2}$, the interface velocity is $v_{n}=h_{t} /\left[1+h_{x}^{2}\right]^{1 / 2}$, and the surface gradient of the temperaturedependent surface energy $\gamma=\gamma(T)$ is given by

$$
\nabla_{S} \gamma=\gamma_{T} \frac{\left(T_{x}+h_{x} T_{y}\right)}{\sqrt{1+h_{x}^{2}}} \mathbf{t}
$$

where $\gamma_{T}=d \gamma / d T$ and $\mathbf{t}$ is the unit tangent vector to the interface in the direction of increasing $x$. Here $h_{x x}$ indicates the second derivative of $h$, etc.

\subsubsection{Inert System}

For an inert system the interface is a material surface, so that we have

$$
\begin{aligned}
& \mathbf{u}^{\alpha} \cdot \mathbf{n}=v_{n}, \\
& \mathbf{u}^{\beta} \cdot \mathbf{n}=v_{n} .
\end{aligned}
$$

The continuity of heat flux gives

$$
\llbracket k \frac{\partial T}{\partial n} \rrbracket=0,
$$

where $k$ is the thermal conductivity and $\partial T / \partial n=\left(T_{y}-h_{x} T_{x}\right) / \sqrt{1+h_{x}^{2}}$ is the normal derivative of the temperature field in each phase.

\subsubsection{Two-Phase System}

Mass conservation across the interface takes the form

$$
\llbracket \rho\left(\mathbf{u} \cdot \mathbf{n}-v_{n}\right) \rrbracket=0 .
$$


Thermodynamic equilibrium at the interface is given by

$$
\llbracket g_{m}(T, p) \rrbracket=0,
$$

where $g_{m}(T, p)$ is the Gibbs free energy density, with $d g_{m}=-s_{m} d T+d p / \rho$, and $s_{m}$ is the entropy density. The continuity of flux gives

$$
\llbracket k \frac{\partial T}{\partial n} \rrbracket=\llbracket \rho\left(\mathbf{u} \cdot \mathbf{n}-v_{n}\right) h_{m} \rrbracket,
$$

where $h_{m}$ is the enthalpy density. Using Eq. (2.12), this can be written in the form

$$
\llbracket k \frac{\partial T}{\partial n} \rrbracket=\rho_{\alpha}\left(\mathbf{u}^{\alpha} \cdot \mathbf{n}-v_{n}\right) L_{\alpha \beta},
$$

where $L_{\alpha \beta}=h_{m}^{\alpha}-h_{m}^{\beta}$ is the difference in enthalpy density between the phases.

\subsection{Base State}

We linearize about a quiescent base state (also indicated by bars). The thermal field is

$$
\bar{T}^{\alpha}(z)=T_{E}+G_{\alpha} z
$$

in the $\alpha$ phase, and

$$
\bar{T}^{\beta}(z)=T_{E}+G_{\beta} z
$$

in the $\beta$ phase, where $T_{E}$ is the unperturbed interface temperature. The temperature gradients in the base state satisfy

$$
0=k_{\alpha} G_{\alpha}-k_{\beta} G_{\beta} .
$$

The pressure field in the base state is hydrostatic, with

$$
0=\frac{d \bar{p}^{\alpha}}{d z}=-\bar{\rho}_{\alpha} g, \quad 0=\frac{d \bar{p}^{\beta}}{d z}=-\bar{\rho}_{\beta} g .
$$

\subsection{Dimensionless Parameters and Governing Equations}

Following the treatment by Zeren \& Reynolds (1972), we make the equations dimensionless based on a length scale given by the total depth $d=H_{\alpha}+H_{\beta}$, a time scale based on the thermal time $d^{2} / \kappa_{\beta}$, a velocity scale $\kappa_{\beta} / d$, a temperature scale $G_{\beta} d$, and a pressure scale $\nu_{\beta} \kappa_{\beta} \bar{\rho}_{\beta} / d^{2}$. These scales introduce the dimensionless parameters

$$
\begin{gathered}
\nu^{*}=\frac{\nu_{\alpha}}{\nu_{\beta}}, \quad \rho^{*}=\frac{\bar{\rho}_{\alpha}}{\bar{\rho}_{\beta}}, \quad \eta^{*}=\frac{\eta_{\alpha}}{\eta_{\beta}} \\
\kappa^{*}=\frac{\kappa_{\alpha}}{\kappa_{\beta}}, \quad k^{*}=\frac{k_{\alpha}}{k_{\beta}}, \quad G^{*}=\frac{G_{\alpha}}{G_{\beta}}, \quad \mu^{*}=\frac{\mu_{\alpha}}{\mu_{\beta}}, \\
\operatorname{Pr}=\frac{\nu_{\beta}}{\kappa_{\beta}}, \quad \mathrm{Ra}=\frac{g \eta_{\beta} G_{\beta} d^{4}}{\nu_{\beta} \kappa_{\beta}}, \quad \mathrm{Cr}=\frac{\mu_{\beta} \kappa_{\beta}}{d \gamma}, \quad \mathrm{Bo}=\frac{g \rho_{\beta} d^{2}}{\gamma}, \quad \mathrm{Ma}=-\frac{\gamma_{T} G_{\beta} d^{2}}{\mu_{\beta} \kappa_{\beta}},
\end{gathered}
$$

and the geometrical parameter $\ell=-H_{\beta} / H_{\alpha}$. For the two-phase system, we have two additional parameters,

$$
\mathcal{L}_{\alpha \beta}=\frac{\rho_{\beta} L_{\alpha \beta} \kappa_{\beta}}{k_{\beta} G_{\beta} d}, \quad \mathcal{S}_{\alpha \beta}=\frac{s_{\alpha \beta} G_{\beta} d^{3}}{\nu_{\beta} \kappa_{\beta}},
$$

where $s_{\alpha \beta}=s_{m}^{\alpha}-s_{m}^{\beta}$ is the difference in entropy density between the phases, so that $\mathcal{L}_{\alpha \beta}$ and $\mathcal{S}_{\alpha \beta}$ represent a dimensionless latent heat and dimensionless entropy difference, respectively (see table I). Here We note that $\mu^{*}=\rho^{*} \nu^{*}$ and $k^{*} G^{*}=1$. 
We assume a horizontal wavenumber $a$ and a temporal growth rate $\sigma=\sigma_{r}+i \sigma_{i}$. The perturbed quantities (indicated by tildes) then satisfy

$$
\begin{gathered}
i a \tilde{u}^{\alpha}+\tilde{w}_{z}^{\alpha}=0 \\
\operatorname{Pr}^{-1} \sigma \tilde{u}^{\alpha}+i a \tilde{p}^{\alpha} / \rho^{*}=\nu^{*}\left(\tilde{u}_{z z}^{\alpha}-a^{2} \tilde{u}^{\alpha}\right), \\
\operatorname{Pr}^{-1} \sigma \tilde{w}^{\alpha}+\tilde{p}_{z}^{\alpha} / \rho^{*}=\nu^{*}\left(\tilde{w}_{z z}^{\alpha}-a^{2} \tilde{w}^{\alpha}\right)+\eta^{*} \operatorname{Ra} \tilde{T}^{\alpha}, \\
\sigma \tilde{T}^{\alpha}+G^{*} \tilde{w}^{\alpha}=\kappa^{*}\left(\tilde{T}_{z z}^{\alpha}-a^{2} \tilde{T}^{\alpha}\right),
\end{gathered}
$$

for $z>0$, and

$$
\begin{gathered}
i a \tilde{u}^{\beta}+\tilde{w}_{z}^{\beta}=0, \\
\operatorname{Pr}^{-1} \sigma \tilde{u}^{\beta}+i a \tilde{p}^{\beta}=\tilde{u}_{z z}^{\beta}-a^{2} \tilde{u}^{\beta}, \\
\operatorname{Pr}^{-1} \sigma \tilde{w}^{\beta}+\tilde{p}_{z}^{\beta}=\tilde{w}_{z z}^{\beta}-a^{2} \tilde{w}^{\beta}+\operatorname{Ra} \tilde{T}^{\beta}, \\
\sigma \tilde{T}^{\beta}+\tilde{w}^{\beta}=\tilde{T}_{z z}^{\beta}-a^{2} \tilde{T}^{\beta},
\end{gathered}
$$

for $z<0$. Henceforth all variables are considered to be dimensionless.

The boundary conditions at $z=0$ are

$$
\begin{gathered}
\tilde{T}^{\alpha}+G^{*} \tilde{h}=\tilde{T}^{\beta}+\tilde{h}, \\
\tilde{u}^{\alpha}-\tilde{u}^{\beta}=0, \\
\left(\tilde{p}^{\alpha}-\tilde{p}^{\beta}\right)-\operatorname{BoCr}^{-1}\left(\rho^{*}-1\right) \tilde{h}^{2} \operatorname{Cr}^{-1} \tilde{h}=2\left(\mu^{*} \tilde{w}_{z}^{\alpha}-\tilde{w}_{z}^{\beta}\right) . \\
\left(\mu^{*} \tilde{u}_{z}^{\alpha}-\tilde{u}_{z}^{\beta}\right)+i a\left(\mu^{*} \tilde{w}^{\alpha}-\tilde{w}^{\beta}\right)-i a \operatorname{Ma}\left(\tilde{T}^{\alpha}+G^{*} \tilde{h}\right)=0 .
\end{gathered}
$$

\subsubsection{Inert System}

For the inert system, the remaining dimensionless boundary conditions are

$$
\begin{gathered}
\tilde{w}^{\alpha}=\sigma \tilde{h}, \\
\tilde{w}^{\beta}=\sigma \tilde{h}, \\
k^{*} \tilde{T}_{z}^{\alpha}=\tilde{T}_{z}^{\beta} .
\end{gathered}
$$

\subsubsection{Two-Phase System}

For the two-phase system, the remaining dimensionless boundary conditions are

$$
\begin{gathered}
\mathcal{S}_{\alpha \beta}\left[\tilde{T}^{\alpha}+G^{*} \tilde{h}\right]=\tilde{p}^{\alpha} / \rho^{*}-\tilde{p}^{\beta}, \\
\sigma\left(\rho^{*}-1\right) \tilde{h}=\rho^{*} \tilde{w}^{\alpha}-\tilde{w}^{\beta}, \\
k^{*} \tilde{T}_{z}^{\alpha}-\tilde{T}_{z}^{\beta}=\rho^{*} \mathcal{L}_{\alpha \beta}\left(\tilde{w}^{\alpha}-\sigma \tilde{h}\right) .
\end{gathered}
$$

\subsubsection{Control Parameter}

Critical conditions are often determined experimentally by varying the temperature gradient across the system. The temperature gradient $G_{\beta}$ appears in the dimensionless parameters $\mathrm{Ma}, \mathrm{Ra}, \mathcal{L}_{\alpha \beta}$, and $\mathcal{S}_{\alpha \beta}$. In order to study various combinations of the independent effects of buoyancy, latent heat, entropy, and the temperature dependence of the surface energy (Marangoni effect), it is convenient to introduce a separate dimensionless temperature gradient

$$
\mathcal{G}=\frac{G_{\beta} d}{T_{E}}
$$


that can be used as a control parameter. We then introduce versions of the parameters Ma, Ra, $\mathcal{L}_{\alpha \beta}$, and $\mathcal{S}_{\alpha \beta}$ that are independent of $G_{\beta}$ by setting

$$
\mathrm{Ma}=\widetilde{\operatorname{Ma}} \mathcal{G}, \quad \operatorname{Ra}=\widetilde{\operatorname{Ra}} \mathcal{G}, \quad \mathcal{L}_{\alpha \beta}=\widetilde{\mathcal{L}}_{\alpha \beta} / \mathcal{G}, \quad \mathcal{S}_{\alpha \beta}=\widetilde{\mathcal{S}}_{\alpha \beta} \mathcal{G}
$$

where

$$
\widetilde{\mathrm{Ma}}=-\frac{\gamma_{T} T_{E} d}{\mu_{\beta} \kappa_{\beta}}, \quad \widetilde{\mathrm{Ra}}=\frac{g \eta_{\beta} T_{E} d^{3}}{\nu_{\beta} \kappa_{\beta}}, \quad \widetilde{\mathcal{L}}_{\alpha \beta}=\frac{\rho_{\beta} L_{\alpha \beta} \kappa_{\beta}}{k_{\beta} T_{E}}, \quad \widetilde{\mathcal{S}}_{\alpha \beta}=\frac{s_{\alpha \beta} T_{E} d^{2}}{\nu_{\beta} \kappa_{\beta}} .
$$

Linear stability calculations are then performed by varying $\mathcal{G}$ for fixed values of $\widetilde{\mathrm{Ma}}, \widetilde{\mathrm{Ra}}$, $\widetilde{\mathcal{L}}_{\alpha \beta}$, and $\widetilde{\mathcal{S}}_{\alpha \beta}$; these parameters then can be set to zero to consider simplified systems. We note that Zeren \& Reynolds introduce the parameter

$$
\Gamma=\frac{\mathrm{Ra}}{\mathrm{Ma}}=\frac{-\rho g \eta_{\beta} d^{2}}{\gamma_{T}}
$$

which is also independent of the temperature gradient, and perform calculations by varying Ma for a fixed value of $\Gamma$.

\section{Numerical Implementation}

We solve the eigenvalue problem that governs the linear stability of the system by using two complementary procedures. In the first approach, the equations are discretized using pseudo-spectral Chebyshev collocation, and the resulting generalized matrix eigenvalue problem is solved using the package RGG from the EISPACK software library (Smith et al. 1976). For a discretization with $N$ degrees of freedom, this routine produces approximations to the first $N$ eigenvalues of the system. The second approach is to use the two-point boundary value solver BVSUP (Scott \& Watts 1977), coupled with the root finder SNSQ (Powell 1970), both from the SLATEC library (Fong et al. 1993), to implement a method described by Keller (1976) to solve the eigenvalue problem. The BVSUP procedure provides a very accurate solution for a given eigenmode provided a good enough initial estimate is available for the root-finding procedure. The pseudospectral method is efficient for small values of $N$, and is well-suited for searching parameter space to detect real and complex eigenvalues. Rather than performing fine grid calculations with the pseudospectral procedure, however, the coarse grid results from the pseudospectral method are often used as initial guesses for the BVSUP code. Continuation from previous solutions is also used once an eigenmode has been identified.

The BVSUP software works in a single domain, so we have mapped the two layers to a common domain by setting

$$
\bar{z}=\left\{\begin{array}{cc}
z & \text { for } 0<z<H_{\alpha}, \\
-H_{\alpha} z / H_{\beta} & \text { for }-H_{\beta}<z<0,
\end{array}\right.
$$

so that $0<\bar{z}<H_{\alpha}$ in each phase. We then have

$$
\frac{d}{d z}=\left\{\begin{array}{cc}
d / d \bar{z} & \text { for } 0<z<H_{\alpha} \\
(1 / \ell) d / d \bar{z} & \text { for }-H_{\beta}<z<0
\end{array}\right.
$$

where $\ell=-H_{\beta} / H_{\alpha}$. To simplify the treatment of the problem, we also introduce an auxiliary ordinary differential equation in $\bar{z}$ for the interface $\tilde{h}$, by setting

$$
\frac{d \tilde{h}}{d \bar{z}}=0,
$$


which allows us to avoid eliminating $\tilde{h}$ as an unknown from the interface boundary conditions.

\section{Results}

In this section we present numerical results for the linear stability of the two-layer system. We first provide a comparison with previous results of Zeren \& Reynolds (1972), who consider an inert system consisting of benzene overlying water, to validate the numerical procedure. We then compute results for a water-steam system, emphasizing the effects associated with the change of phase. These results are contrasted with those for inert layers with thermophysical properties corresponding to the water-steam system.

\subsection{Comparison with Zeren and Reynolds}

In order to validate the numerical procedure, we have reproduced and extended the numerical results of Zeren \& Reynolds (1972). We use the thermophysical parameters given in their Table II for a temperature of $16^{\circ} \mathrm{C}$. We consider their case $d_{b}^{*}=H_{\beta} /\left(H_{\alpha}+\right.$ $\left.H_{\beta}\right)=0.4$. Zeren and Reynolds consider both the case of heating from above (positive $\mathrm{Ma}$ ), where the main driving force is the Marangoni effect, and buoyancy effects are expected to be stabilizing, and the case of heating from below (negative Ma), where both buoyancy effects and Marangoni effects can produce instabilities.

If the system is heated from above, Zeren and Reynolds compute a positive critical Marangoni number of 1486 , with a critical wavenumber of $a=2.6$; they consider only stationary modes with $\sigma_{i}=0$. For $a=2.6$ we find $\mathrm{Ma}=1468.4635$, in fair agreement with their results. We find a critical wavenumber to three digits of $a=2.66$, with $\mathrm{Ma}=$ 1466.8951. Ferm \& Wollkind (1982) also obtained similar agreement in a comparison with Zeren and Reynolds, although they chose different thermophysical properties for the benzene-water system.

Our computed neutral stability curves for this case are shown in Fig. 2. We show the two stationary modes that are given in Fig. 2 of Zeren and Reynolds; the convection pattern in these modes has a single cell in each layer, with negligible surface deformation. The convection is more concentrated near the interface for the higher wavenumber mode. We also find an oscillatory mode $\left(\sigma_{i} \neq 0\right)$ at intermediate wavenumbers and Marangoni numbers. As discussed by Zeren and Reynolds, the critical mode is mainly driven by Marangoni convection. The oscillatory mode has a minimum near $a=5.5$, with $\mathrm{Ma}=$ 16859.007 and $\sigma_{i}= \pm 123.7$. The surface deflection for this mode is also negligible.

If the system is heated from below, Zeren and Reynolds compute a negative critical Marangoni number of $\mathrm{Ma}=-6068$, with a critical wavenumber of $a=9$. For $a=9.0$ we find $\mathrm{Ma}=-6014.4082$. We show our computed marginal stability curves for this case in Fig. 3. We find that the critical mode is oscillatory, with Ma $=-2849.5156$ with $a=4.5$ and $\sigma_{i}= \pm 56.82$. The surface deflection for this mode is negligible. This mode merges with another stationary branch that has a long-wavelength asymptote; $\sigma_{i}$ tends to zero on the oscillatory branch as the modes merge. On the long-wavelength branch, for $a=0.001$ we find $\mathrm{Ma} \approx-19318.282$. Analytic results for long-wavelength modes are described in the Appendix; for this case the asymptotic result is $\mathrm{Ma}=-19318.06$, in excellent agreement with the numerical results.

There is a single convection cell in each layer for the long wavelength mode, with a significant interface deflection. Examination of the eigenmode shows that there is downflow in the layer beneath the elevation. As discussed by Scriven \& Sternling (1964) for the single layer case, Marangoni modes and buoyant modes can be distinguished by the direction of the vertical flow in the layer beneath an interface elevation: there is downflow beneath 
elevations for Marangoni flow, and upflow beneath elevations for buoyancy-driven flow. This is consistent with the Zeren and Reynold's interpretation of the long-wavelength mode as driven by Marangoni effects. The other two stationary modes in Fig. 3 with minima near $a=10$ have negligible surface deflection. For both modes the lower layer is uni-cellular and nearly isothermal, and the upper layer exhibits two vertically-stacked convective cells for the mode with $\mathrm{Ma} \approx-6,000$, and three vertically-stacked convective cells for the mode with $\mathrm{Ma} \approx-50,000$.

\subsection{Water-Steam System}

We next consider parameters appropriate for a water-steam system Wagner (1998). The water-steam system has a critical point at temperature $T=647.096 \mathrm{~K}$, and we choose parameters at the lower temperature $T=640 \mathrm{~K}$, which is far enough below the critical point to allow an incompressible approximation for the flow field to be valid. Dimensional parameter values are given in Table I, and corresponding dimensionless parameters are given in Table II.

\subsubsection{Inert System}

We begin with computations for an idealized inert system with thermophysical parameters appropriate to the water-steam system, followed by a comparison to the results for the actual two-phase system. Our calculations correspond to a system with steam overlying water, with a depth ratio of $H_{\alpha} / H_{\beta}=2$.

In Fig. 4 we show marginal stability curves for an inert water-steam system heated from above. The top two curves in the figure represent a stationary mode (solid curve) and an oscillatory mode (dashed curve); for these conditions the most dangerous mode is stationary with a critical wavenumber of $a=42.0$ and $\mathrm{Ma}=289278.34$. The stationary mode does not extend to low wavenumbers, but is joined by an oscillatory mode that has a nearby minimum at $a=17.0$, with $\mathrm{Ma}=383756.76$ and $\sigma_{i}= \pm 1624.6$. The oscillatory mode also exhibits a higher relative minimum at a lower wavenumber. The lower solid curve in Fig. 4 shows the marginal stability curve for $\Gamma=0$. As might be expected for heating from above, the system is seen to be destabilized significantly if buoyancy is eliminated, with a critical Marangoni number of $\mathrm{Ma}=809.52$ and wavenumber $a=3.37$ for this stationary mode. The interface deflection is negligible for these modes; making the interface non-deformable by setting $\mathrm{Cr}=0$ does not change the minima in these curves to graphical accuracy. There is uni-cellular convection in each layer for these modes. The large wavenumber behavior of the stationary modes can be obtained through an approximate analysis, as outlined in the Appendix; these results are shown as the solid points in Fig. 4. The large wavenumber analysis assumes that $\Gamma=0$ (which eliminates the effects of buoyancy) and $\mathrm{Cr}=0$ (which eliminates interface deformation). The large wavenumber results are seen to be in good agreement with the numerical results for $\mathrm{Cr}=1.12 \cdot 10^{-6}$ and for both $\Gamma=5.24$ and $\Gamma=0$. The neutral curve for $\Gamma=0$ rises to an asymptote near $a=0.03$ and does not extend to smaller wavenumbers (see below).

The case of heating from below is more complicated because both buoyancy and the Marangoni effect are destabilizing; we consider several special cases to help interpret the results.

In Fig. 5 we show marginal stability curves for an inert water-steam system heated from below with buoyancy neglected $(\Gamma=0)$. The most dangerous mode is oscillatory, with a critical wavenumber $a=4.2$ for $\mathrm{Ma}=-35784.61$ and $\sigma_{i}= \pm 237.07641$. The flow is uni-cellular in each layer for this mode, and the interface deformation is insignificant; graphically identical results are obtained by setting $\mathrm{Cr}=0$. The oscillatory mode merges with a stationary mode near $a=0.025$, where $\sigma_{i} \rightarrow 0$. The stationary mode has a 
small-wavenumber limit that is given approximately by the expression

$$
-\mathrm{Ma}=\frac{3.63 \times 10^{6}+1.12 \times 10^{6} a^{2}}{1-1.34 \times 10^{3} a^{2}},
$$

(see Eq. (8.7) in the Appendix), and these results are shown as the solid dots in Fig. 5. Note that there is a pole in this expression for $a=2.74 \times 10^{-2}$. The low-wavenumber stationary mode has a significant interface deflection, and is sensitive to the value of Cr. The flow is uni-cellular in each layer. There is downflow in the lower layer beneath the interface elevations, consistent with the stationary mode arising from the Marangoni effect. The stationary mode rises to an asymptote near $a=0.03$, which is the same wavenumber as that showing asymptotic behavior in Fig. 4. In fact, combining the results for the stationary modes with $\Gamma=0$ in Fig. 4 and Fig. 5 by plotting instead 1/Ma versus $a$ produces a single smooth curve crossing the $\mathrm{y}$-axis with $1 / \mathrm{Ma}=0$ at a wavenumber $a=0.0274$.

We next set $\widetilde{\mathrm{Ma}}=0$ to eliminate the Marangoni effect, so that in the system heated from below the resulting modes are due solely to buoyancy. In Fig. 6 we show a number of neutral modes plotted as a function of $-\mathrm{Ra} / \Gamma$ versus wavenumber with $\Gamma=5.24$ as before; this retains the scaling on the ordinate to facilitate comparison with the previous figures where $\mathrm{Ma}=\mathrm{Ra} / \Gamma$. The most dangerous mode is stationary with a critical wavenumber $a=4.2$ for $\mathrm{Ra} / \Gamma=-478.7587$. Most of the flow for this mode is confined to a single cell in the upper layer, and there is negligible interface deflection. This mode extends to small wavenumbers, and the small-wavenumber analysis predicts an asymptotic value $\mathrm{Ra} / \Gamma=-2.727 \times 10^{7}$, which is in good agreement with the numerical results. The two-layer system supports a number of buoyant modes for each wavenumber, and each mode can be viewed to a good approximation as concentrated in either the upper or lower layer, while differing in the number of cells that are stacked vertically within the layer. Indeed, if the interface is replaced by a rigid, isothermal boundary, there are two uncoupled families of modes which are each entirely confined to a single layer. These two families have intersecting neutral curves, depending in detail on the material properties and the layer depths. With the actual boundary conditions appropriate to a conducting two-fluid interface, these modes are weakly coupled, and there is a strong flow in one layer and a weak flow in the other. Because of the coupling, the points where neutral curves cross in the uncoupled case are modified so that the curves either avoid each other near these points or are connected by short regions of oscillatory behavior; both types of behavior can be seen in Fig. 6. At a wavenumber of $a=4$, the first few modes in increasing order of $-\mathrm{Ra} / \Gamma$ shown in Fig. 6 can be denoted as $\mathrm{U}_{1}, \mathrm{U}_{2}, \mathrm{U}_{3}, \mathrm{~L}_{1}, \mathrm{U}_{4}$, and $\mathrm{L}_{2}$, where $\mathrm{U}_{1}$ denotes a mode with a single cell in the upper layer, $\mathrm{L}_{2}$ denotes a mode with two vertically-stacked cells in the lower layer, and so on. For all of these modes, there is negligible interface deflection, and the modes are primarily stationary, except where two nearby modes are coupling. The mode crossings become quite complicated for larger wavenumbers, where the neutral modes are all accumulating near a common asymptotic behavior.

In Fig. 7 we show neutral stability curves for the inert case including both buoyancy and Marangoni effects. A number of stationary and oscillatory modes occur, although the lowest mode is stationary with a critical wavenumber of $a=5.4$ for $\mathrm{Ma}=-895.23$. There is negligible interface deflection at this wavenumber. The flow is concentrated in the upper layer, primarily with a single cell over most of the layer that is accompanied by a second weaker cell in the vicinity of the interface. This mode extends to small wavenumbers, and the small-wavenumber analysis predicts an asymptotic value $\mathrm{Ma}=-3.203 \cdot 10^{6}$, which is in good agreement with the numerical results. At small wavenumbers this mode 
shows significant interface deflection, with uni-cellular flow in each layer and downflow in the lower layer beneath the interface deflections, consistent with a Marangoni flow. At intermediate wavenumbers between these regions there is an oscillatory branch that at each end terminates on the stationary mode, and lies slightly below it. There are also higher oscillatory modes with well-defined minima that are interspersed with higher stationary modes. The oscillatory modes occasionally cross the stationary modes, and cause distortions in the stationary neutral curves at these points. As in Fig. 6, there is an accumulation of neutral curves at larger wavenumbers. The lower neutral curves are primarily buoyant modes that are modified by the Marangoni effect, which is stabilizing in this case (c.f. Fig. 6). The remnant of the stationary Marangoni mode that occurs at small wavenumbers in Fig. 5 is also evident in Fig. 7 at these wavenumbers, but in Fig. 7 the coupling with buoyancy has broken this mode into a number of disconnected branches where the mode intersects with the longer wavenumber buoyant modes. For example, at a wavenumber of $a=0.2$, the stationary mode for $\mathrm{Ma}=-8.702 \cdot 10^{6}$ shows a significant interface deflection, suggesting that there is a sizable Marangoni component to the flow.

\subsubsection{Two-Phase System}

We next consider the stability of a two-phase system using the interfacial boundary conditions appropriate to the description of a phase transition. This introduces the additional parameters $\widetilde{\mathcal{S}}_{\alpha \beta}$ and $\widetilde{\mathcal{L}}_{\alpha \beta}$ representing the jumps in entropy and enthalpy across the interface. In our calculations we find that the stability of the system is quite sensitive to these parameters, and in fact the nature of the driving mechanism changes as the entropy varies from small to large values. We therefore have included calculations performed for various values of $\widetilde{\mathcal{S}}_{\alpha \beta}$, while keeping the ratio of $\widetilde{\mathcal{S}}_{\alpha \beta}$ and $\widetilde{\mathcal{L}}_{\alpha \beta}$ fixed at its nominal value for the steam water system under our chosen conditions. We note that in dimensional terms these quantities are related by $L_{\alpha \beta}=T_{E} S_{\alpha \beta}$, where $T_{E}$ is the equilibrium transition temperature at the interface. The value of the dimensionless entropy for the water-steam system is quite large, and tends to mask the appearance of the Marangoni mode, as will be discussed below.

In Fig. 8 we show neutral stability curves for the water-steam system heated from above (Ma $>0$ ) without buoyancy $(\Gamma=0)$ for four values of $\widetilde{\mathcal{S}}_{\alpha \beta}$. For $\widetilde{\mathcal{S}}_{\alpha \beta}=0$, the system is unstable to a stationary mode with a critical wavenumber $a=2.7$ for $\mathrm{Ma}=227.20$. The flow is qualitatively similar to the corresponding inert case with $\Gamma=0$ shown in Fig. 4. The flow is mostly confined to the upper layer and uni-cellular, without significant interface deformation. In contrast to the corresponding mode shown in Fig. 4, this neutral curve exhibits asymptotes at both smaller $\left(a=8.88 \times 10^{-3}\right)$ and larger $(a=7.76)$ wavenumbers; no large wavenumber asymptotic behavior is observed. As the entropy difference $\widetilde{\mathcal{S}}_{\alpha \beta}$ increases, this mode is stabilized, and the wavenumbers of the asymptotes approach each other. For the largest value $\widetilde{\mathcal{S}}_{\alpha \beta}=2.955 \times 10^{8}$ shown in the figure, the range of unstable wavenumbers has contracted to $0.27<a<0.37$, and for slightly larger values of $\widetilde{\mathcal{S}}_{\alpha \beta}$ the system is linearly stable. For the parameter values in Table II (including the case $\Gamma \neq 0$ ) we have found no instabilities in heating from above.

In Fig. 9 we show neutral stability curves for the water-steam system heated from below $(\mathrm{Ma}<0)$ without buoyancy $(\Gamma=0)$ for three values of $\widetilde{\mathcal{S}}_{\alpha \beta}=0,2.955 \times 10^{8}$, and $3.5 \times 10^{8}$; we note that the value of $\widetilde{\mathcal{S}}_{\alpha \beta}$ for the water-steam system in Table II is $\widetilde{\mathcal{S}}_{\alpha \beta}=5.86 \times 10^{14}$. For $\widetilde{\mathcal{S}}_{\alpha \beta}=0$, the marginal stability curve has two stationary branches at high and low wavenumbers. The two branches have asymptotes for $a=8.88 \times 10^{-3}$ and $a=7.76$, which are the same values obtained for the asymptotes of the marginal stability curve 
shown in Fig. 8 for $\widetilde{\mathcal{S}}_{\alpha \beta}=0.0$ with heating from above $(\mathrm{Ma}>0)$. As in the inert case, if the data is instead plotted as 1 /Ma versus wavenumber, a single smooth neutral curve is obtained that passes through the points $1 / \mathrm{Ma}=0$ at the wavenumbers corresponding to the asymptotes occurring in Fig. 8 and Fig. 9 for $\widetilde{\mathcal{S}}_{\alpha \beta}=0$. For heating from below, there is gap in wavenumber between the asymptotes where there is no stationary mode; instead an oscillatory mode is observed that begins and ends with $\sigma_{i}=0$ at points on the stationary branches. The minimum on the $\widetilde{\mathcal{S}}_{\alpha \beta}=0$ neutral curves occurs at a critical wavenumber $a=10.8$ for $\mathrm{Ma}=-4028.83$. Similar behavior also occurs for $\widetilde{\mathcal{S}}_{\alpha \beta}=2.955 \times 10^{8}$, with a critical wavenumber $a=5.4$ for $\mathrm{Ma}=-681.22$. In this case the distance between asymptotes of the stationary modes has deceased considerably, as in Fig. 8. For $\widetilde{\mathcal{S}}_{\alpha \beta}=3.5 \times 10^{8}$, the asymptotes have disappeared, and a single stationary mode occurs over the full range of wavenumbers. The minimum on the neutral curves occurs at a critical wavenumber $a=4.4$ for $\mathrm{Ma}=-478.30$. Although there is no gap in wavenumber for the stationary mode in this case, an oscillatory mode persists over a small range of wavenumbers near $a=0.01$, as can be seen by careful examination of Fig. 9.

For still larger values of $\mathcal{S}_{\alpha \beta}$ than we have considered in Fig. 8 and Fig. 9, we have not found any neutral modes for the case of heating from above, either with or without buoyancy. For the case of heating from below with buoyancy, for values of $\widetilde{\mathcal{S}}_{\alpha \beta}$ and $\widetilde{\mathcal{L}}_{\alpha \beta}$ appropriate for the water-steam system, the neutral curves shown in Fig. 10 are obtained. For this value of $\widetilde{\mathcal{S}}_{\alpha \beta}$ we find that these modes are all insensitive to $\gamma_{T}$, in that setting $\widetilde{\mathrm{Ma}}=0$ does not affect the indicated modes. To emphasize the insensitivity to $\widetilde{\mathrm{Ma}}$, in Fig. 10 we plot results in terms of $\mathcal{G}$ as discussed in Section 2.4.3. (More specifically, we plot $-10^{8} \mathcal{G}$ versus wavenumber, which retains the overall scale used in previous plots of $\mathrm{Ma}=\widetilde{\mathrm{Ma}} \mathcal{G}$ versus wavenumber for $\widetilde{\mathrm{Ma}}=1.004 \times 10^{8}$.) The most dangerous mode occurs at low wavenumbers, and is found to be insensitive to buoyancy as well; this is a novel two-phase mode that we discuss in more detail below. The remainder of the modes shown in Fig. 10 are all buoyant modes that are qualitatively similar to those shown in Fig. 7. There are oscillatory modes in Fig. 10 connecting regions where the low-wavenumber mode intersects the family of stationary buoyant modes; the oscillatory modes occur over very small ranges of wavenumber and are barely visible in the figure. The most dangerous of the buoyant modes has a critical wavenumber $a=4.5$ for $10^{8} \mathcal{G}=-527.19$, with uni-cellular flow mainly in the top layer and no significant interface deformation.

For the parameter values in Table II the low-wavenumber mode is insensitive to the Marangoni effect and, for low wavenumbers, to the effects of buoyancy. It is, however, sensitive to the entropy $\widetilde{\mathcal{S}}_{\alpha \beta}$ and latent heat $\widetilde{\mathcal{L}}_{\alpha \beta}$, and is the continuation of the Marangoni mode shown in Fig. 9 for $\widetilde{\mathcal{S}}_{\alpha \beta}=3.5 \times 10^{8}$ to much larger values of $\widetilde{\mathcal{S}}_{\alpha \beta}$. In the process, the critical wavenumber has decreased significantly, and the Marangoni effect has been rendered ineffective by the large entropy. This can be seen from the boundary conditions given in Eq. (2.35) and Eq. (2.39). For large values of $\widetilde{\mathcal{S}}_{\alpha \beta}$, Eq. (2.39) shows that the interface becomes isothermal, and the Marangoni term in Eq. (2.35) that is proportional to the perturbed interface temperature is then negligible.

This mode is shown in more detail for smaller wavenumbers in Fig. 11. In these calculations we have eliminated the effects of buoyancy by setting $\Gamma=0$. To help understand this mode, we have also performed computations that illustrate the effects of the Bond number Bo and the Crispation number $\mathrm{Cr}$ for this mode. Three cases are shown in Fig. 11; the case corresponding to Table II is the intermediate curve and data points that asymptote to a small wavenumber limit for $10^{8} \mathcal{G}=-2.8$. The upper curve and data points result from setting $\mathrm{Cr}=0$, and the lower curve and data points correspond 
to setting $\mathrm{Bo}=0$. The solid curves correspond to numerical results, and the symbols on the curves correspond to analytical results from a small-wavenumber approximation given in the Appendix. The small-wavenumber results depend strongly on both Bo and Cr. For $\mathrm{Cr} \neq 0$ and $\mathrm{Bo} \neq 0$,

$$
-10^{8} \mathcal{G} \approx \frac{d_{1}\left[1-\rho^{*}\right] \text { Bo }}{d_{2} \mathrm{Cr}}=2.8351
$$

For $\mathrm{Cr}=0$

$$
-10^{8} \mathcal{G} \approx \frac{-d_{1}}{d_{4}} a^{-2}-\frac{d_{6}}{d_{4}}=\frac{2.7668 \times 10^{6}}{a^{2}}+3.4724 \times 10^{6},
$$

For $\mathrm{Bo}=0$ and $\mathrm{Cr} \neq 0$

$$
-10^{8} \mathcal{G} \approx \frac{d_{1}}{d_{2} \mathrm{Cr}} a^{2}=0.1925 a^{2} .
$$

Here the coefficients $d_{1}, d_{2}, d_{4}$, and $d_{6}$ depend on the layer geometry and the remaining material constants and are given in the Appendix; here we have evaluated these expressions for the values given in Table II. All three curves have the same behavior for larger wavenumbers, given by a large wavenumber approximation shown as the dashed curve. As described in the Appendix, this curve is approximately given by

$$
-10^{8} \mathcal{G} \approx 1.0097 \times 10^{6} a .
$$

\subsubsection{Rayleigh-Taylor Instability}

The two-layer system can also exhibit the classical Rayleigh-Taylor instability if heavier fluid overlies lighter fluid. In the absence of buoyancy effects the gravitational stability of the system is governed by a simple potential energy argument that balances the increased surface energy of a deformed interface $y=h(x)$ against the change in the gravitational potential energy of the displaced fluid,

$$
g\left(\bar{\rho}_{\alpha}-\bar{\rho}_{\beta}\right) h=-\gamma h_{x x}
$$

In terms of our dimensionless variables, this takes the simple form

$$
-\mathrm{Bo}=\frac{a^{2}}{\left(1-\rho^{*}\right)},
$$

which can be seen as a factor in the normal stress balance boundary condition (2.34).

In the situation we have studied above, we have lighter steam overlying heavier water, so the Rayleigh-Taylor instability does not occur. To study this instability for our system with a minimal change in notation, we temporarily choose to change the direction of gravity while keeping steam and water in the original orientation, so that the water and steam are unstably stratified with respect to gravity. We take $G_{\beta}>0$, so that buoyancy has a stabilizing effect on the system; the resulting sign conventions produce $\mathrm{Ma}<0$, Ra $>0, \Gamma<0$, and Bo $<0$. In Fig. 12 we show the corresponding numerical results for the Rayleigh-Taylor instability. The dashed curve in Fig. 12 shows the curve $-\mathrm{Bo}=a^{2} /\left(1-\rho^{*}\right)$ that holds for $\Gamma=0$. The solid curve shows numerical marginal stability results for $\mathrm{Ma}=-1.0 \times 10^{6}$ and $\Gamma=-5.24$. The stabilizing effects of buoyancy are evident at small wavenumbers, where the system is then stable if $|\mathrm{Bo}|$ is sufficiently small.

\section{Discussion}

A comparison of instabilities for the inert and two-phase cases can be made in the large wavenumber limit that is summarized in the Appendix. To make this comparison 
we ignore buoyancy by setting $\Gamma=0$, and also ignore interface deformation by setting $\mathrm{Cr}=0$. To help visualize the flow we introduce a two-dimensional streamfunction $\psi$ with $w=\psi_{x}$ and $u=-\psi_{z}$. The corresponding eigenfunction is given in the Appendix for each case.

For the inert case contours of the temperature and streamfunction are shown in Fig. 13. Here we have exaggerated the size of the perturbation to emphasize the distortion of the isotherms near the interface. To make the plots easier to interpret we have assumed equal thermal conductivities $\left(k^{*}=1\right)$, which makes the unperturbed temperature gradients in the two layers the same and facilitates comparison of the perturbed temperature fields in each layer. There is a temperature gradient along the interface, and the streamlines of the flow are along the interface. Over the single period of the flow shown in Fig. 13, there are four flow cells near the interface that alternately compress and expand the isotherms near the interface. For our parameters with $\kappa^{*}<1$, the Marangoni instability occurs for heating from above $(\mathrm{Ma}>0)$ in the large wavenumber limit. If $\kappa^{*}>1$ the instabilities occur for heating from below instead.

The driving mechanism for the large-wavenumber instability of the two-phase system arises from the coupling of the temperature field and vertical velocity that occurs in the thermal transport equations (2.27) and (2.31) and the latent heat boundary condition (2.41). The coupling is illustrated in the contours of the temperature and streamfunction for the two-phase system case as shown in Fig. 14. Here to simplify the plot we have assumed the material properties are equal in both phases, and have exaggerated the size of the perturbation to emphasize the distortion of the isotherms near the interface. For the large value of $\mathcal{S}_{\alpha \beta}$ in the steam-water system there is no significant interface deformation. The fluid flow is normal to the interface, and the plot shows that the downflow in the center of the plot tends to compress the distance between isotherms near the interface in the upper $(\alpha)$ phase, and expand the distance between them in the lower phase. The opposite is true for the regions with upflow at the interface. The resulting net change in the temperature fluxes at the interface are balanced by the evolution of latent heat at the interface, which in turn is driven by the vertical velocity at the interface. When the system is heated from below as shown, these effects reinforce each other to drive the instability. When the system is heated from above, the effects are in opposing directions and no instability is possible.

The instability illustrated in Fig. 14 corresponds to the case of large entropy $\mathcal{S}_{\alpha \beta}$, and the Marangoni effect is negligible in this limit. For very small values of $\mathcal{S}_{\alpha \beta}$, the Marangoni effect become dominant and produces a large-wavenumber instability that is qualitatively similar to the inert case shown in Fig. 13.

If the bilayer is heated from below, in the rest state the upper layer of gas is colder than the equilibrium temperature and the lower layer of liquid is hotter than the equilibrium temperature; these are both potentially destabilizing distributions. The pressure in the upper layer of gas is lower than the equilibrium pressure and the pressure in the lower layer of liquid is higher than the equilibrium pressure, which tends to stabilize the system. As discussed in the introduction, a criterion for the underlying liquid ( $\beta$ phase) to be superheated is obtained by comparing the local pressure and temperature gradients at the interface with the Clausius-Clapeyron equation (1.1), giving

$$
-G_{\beta}>\frac{g\left(\bar{\rho}_{\beta} / \bar{\rho}_{\alpha}-1\right)}{\left(s_{m}^{\alpha}-s_{m}^{\beta}\right)} .
$$


Similarly, the criterion for the overlying gas ( $\alpha$ phase) to be supercooled is

$$
-G_{\alpha}>\frac{g\left(1-\bar{\rho}_{\alpha} / \bar{\rho}_{\beta}\right)}{\left(s_{m}^{\alpha}-s_{m}^{\beta}\right)} .
$$

If we assume $\widetilde{\mathrm{Ra}}=0$ and $\widetilde{\mathrm{Ma}}=0$ in the small-wavenumber expansion given in Eq. (8.15) we obtain the dimensional result

$$
-G_{\beta}=\left[\frac{\rho^{*} d_{1}}{d_{2}^{(2)}}\right] \frac{g\left(\bar{\rho}_{\beta} / \bar{\rho}_{\alpha}-1\right)}{\left(s_{m}^{\alpha}-s_{m}^{\beta}\right)},
$$

where the prefactor evaluates to $\rho^{*} d_{1} / d_{2}^{(2)}=0.465$ for our system. Thus a supercooling argument is in qualitative agreement with the observed low-wavenumber instability. The supercooling argument is not expected to be quantitatively accurate, since it neglects the effects of capillarity and of flow, which arise when there are lateral pressure and temperature gradients in the system.

\section{Conclusions}

We have performed linear stability calculations for horizontal fluid bilayers that can undergo a phase transformation, taking into account both buoyancy effects and thermocapillary effects. We compare the familiar case of the stability of two immiscible fluids in a bilayer geometry (the "inert" case) with the less-studied case that the two fluids represent different phases of a single-component material. The two cases differ in their interfacial boundary conditions; the two equations expressing the fact that the interface is an impenetrable material surface in the inert case are replaced by the simple continuity of mass flux across the interface, together with an assumption of thermodynamic equilibrium that in the linearized equations represents the Clausius-Clapeyron relation relating the interfacial temperature and pressures. In addition, the energy balance contains a term representing the addition of latent heat in the two phase case that is absent in the inert case.

We mainly consider the case of the lighter phase overlying the heavier phase, so that the base state is stably stratified in this sense. We find that, as in the inert case, the two-phase system can be linearly unstable to either heating from above $(\mathrm{Ma}>0)$ or below $(\mathrm{Ma}<0)$. More specifically, for small values of the entropy difference between the

phases, $\widetilde{\mathcal{S}}_{\alpha \beta}$, the marginal stability curve, if plotted as $1 /$ Ma versus the wavenumber $a$, is smooth with both positive and negative values (see Figs. $8 \& 9$ ). The zeroes of this curve then represent wavenumbers for which a plot of Ma versus wavenumber exhibits vertical asymptotes. The two-phase instability persists to small wavenumbers in the case of heating from below. For the larger values of $\widetilde{\mathcal{S}}_{\alpha \beta}$ that characterize the water-steam system, we find that the two-phase system is unstable only for heating from below (see Fig. 10). The large value of $\widetilde{\mathcal{S}}_{\alpha \beta}$ renders the system insensitive to the value of $\gamma_{T}$ for both heating from above and below in the water-steam system, and the Marangoni effect is masked by a stronger effect due to the entropy difference. To help understand the mechanisms driving the instability on heating from below we have performed both longwavelength and short-wavelength analyses of the two-phase system. The short-wavelength analysis (large $a$ ) shows that the instability is driven by a coupling between the flow normal to the interface and the latent heat generation at the interface (see Figs. 13 \& 14). The mechanism for the large wavelength (small $a$ ) instability is more complicated (see Fig. 11). The detailed form of the expansion, viz, the exponent $n$ in the leading order expansion $\mathrm{Ma} \sim a^{n}$ depends on the Crispation and Bond numbers as well as $\widetilde{\mathcal{S}}_{\alpha \beta}$. We 
also note that the two-phase system allows a conventional Rayleigh-Taylor instability if the heavier fluid overlies the lighter fluid (Chen \& Fried 2006); applying a temperature gradient allows a stabilization of the interface (see Fig. 12).

\section{Acknowledgments}

G.B.M. and S.R.C. were supported by the Microgravity Research Division of NASA, and K.F.G and D.L.C. were supported by National Research Council Postdoctoral Fellowships during a portion of this research. The authors are grateful for helpful discussions with D. M. Anderson and J. B. Andrews.

\section{REFERENCES}

Andereck, C. D., Colovas, P. W., Degen, M. M. \& Renardy, Y. Y. 1998 Instabilities in two-layer rayleigh-benard convection: overview and outlook. International Journal of Engineering Science 36, 1451-1470.

Anderson, D. M., Cermelli, P., Fried, E., Gurtin, M. E. \& McFadden, G. B. 2006 General dynamical sharp-interface conditions for phase tranformations in viscous heatconducting fluids. J. Fluid Mech., in press .

Anderson, D. M., McFadden, G. B. \& Wheeler, A. A. 1998 Diffuse-interface methods in fluid mechanics. Annual Review of Fluid Mechanics 30, 139-165.

Anderson, D. M., McFadden, G. B. \& Wheeler, A. A. 2000 A phase-field model of solidification with convection. Physica D 135, 175-194.

Anderson, D. M., McFadden, G. B. \& Wheeler, A. A. 2001 A phase-field model with convection: sharp-interface asymptotics. Physica D 151, 305-331.

Andrews, J. B., Downs, D. A. \& LiU, Q. Q. 2006 Monotectic growth: unanswered questions. Materials Science Forum 508, 45-50.

Busse, F. \& Schubert, G. 1971 Convection in a fluid with two phases. J. Fluid Mech. 46, 801-812.

Busse, F. H. 1989 Fundmanetals of thermal convection. In Mantle Convection: Plate Tectonics and Global Dynamics (ed. W. Peltier), pp. 23-95. Gordon \& Breach.

Callen, H. B. 1960 Thermodynamics. New York: John Wiley \& Sons.

Chen, X. \& Fried, E. 2006 Rayleigh-taylor problem for a liquid-liquid phase interface. submitted to J. Fluid Mech .

Davis, S. H. 1987 Thermocapillary instabilities. Ann. Rev. Fluid Mech. 19, 403-435.

Davis, S. H. 2001 Theory of Solidification. Cambridge, UK: Cambridge University Press.

Edwards, D. A., Brenner, H. \& Wasan, D. T. 1991 Interfacial Transport Processes and Rheology. Boston: Butterworth-Heinemann.

Ferm, E. N. \& Wollkind, D. J. 1982 Onset of Rayleigh-Benard-Marangoni instability: Comparison between theory and experiment. J. Non-Equilib. Thermodyn, 7, 169-190.

Fong, K. W., Jefferson, T. H., Suyehiro, T. \& Walton, L. 1993 Guide to the Slatec common mathematical library. See http://www.netlib.org/slatec/guide. The program SNSQ was written by K. Hiebert and is based on an algorithm of Powell (1970).

Huang, S.-C. \& Glicksman, M. E. 1981 Fundamentals of dendritic solidification - i. steadystate tip growth. Acta Metall. 29, 701-715.

Hunt, J. D. \& Lu, S.-Z. 1994 Crystallisation of eutectics, monotectics, and peritectics. In Handbook of crystal growth 2. Bulk crystal growth, Part B: Growth mechanisms and dynamics (ed. D. T. J. Hurle), pp. 1111-1166. Gordon \& Breach.

Johnson, D. \& NARAYAnAN, R. 1998 Marangoni convection in multiple bounded fluid layers and its application to materials processing. Phil. Trans. R. Soc. Lond. A 356, 885-898.

Joseph, D. D. \& Renardy, Y. Y. 1993 Fundamentals of two-fluid dynamics. Berlin: SpringerVerlag.

Keller, H. B. 1976 Numerical Solution of Two-Point Boundary Value Problems. Philadephia, PA: Society for Industrial and Applied Mathematics, pp. 45-48. 
LANGER, J. S. 1980 Instabilities and pattern formation in crystal growth. Reviews of Modern Physics 52, 1-28.

Mullins, W. W. \& Sekerka, R. F. 1963 Morphological instability of a particle growing by diffusion or heat flow. J. Appl. Physics 34, 323-329.

Mullins, W. W. \& Sekerka, R. F. 1964 Stability of a planar interface during solidifcation of a dilute binary alloy. J. Appl. Physics 35, 444-451.

Nepomnyashchy, A. A., Verlarde, M. G. \& Colinet, P. 2002 Interfacial Phenomena and Convection. London: Chapman \& Hall/CRC.

Parry, W. T., Bellows, J. C., Galligher, J. S. \& Harvey, A. H. 2000 ASME International Steam Tables for Industrial Use. ASME.

Powell, M. J. D. 1970 A hybrid method for nonlinear equations. In Numerical methods for Nonlinear Algebraic Equations (ed. P. Rabinowitz), pp. 87-114. Gordon \& Breach.

Sakurai, S., Tschammer, A., Pesch, W. \& Ahlers, G. 1999 Convection in the presence of a first-order phase change. Phys. Rev. E 60, 539-550.

Schatz, M. F. \& Neitzel, G. P. 2001 Experiments on thermocapillary instabilities. Ann. Rev. Fluid Mech. 33, 93-127.

Scotт, M. R. \& Watts, H. A. 1977 Computational solution of linear two-point boundary-value problems via othonormalization. SIAM J. Numer. Anal. 14, 40-70.

Scriven, L. E. \& Sternling, C. V. 1964 On cellular convection driven by surface-tension gradient: effects of mean surface tension and surface viscosity. J. Fluid Mech. 19, 321-340.

Smith, B. T., Boyle, J. M., Dongarra, J. J., Garbow, B. S., Ikebe, Y., Klema, V. C. \& Moler, C. B. 1976 Matrix Eigenvalue Routines - EISPACK Guide. Springer-Verlag.

StÖcker, C. \& RAtKe, L. 2000 Monotectic composite growth with fluid flow. J. Crystal Growth 212, 324-333.

Tiller, W. A., Jackson, K. A., Rutter, J. W. \& Chalmers, B. 1953 The redistribution of solute atoms during the solidification of metals. Acta Metall. 1, 428-437.

Turner, J. S. 1973 Buoyancy Effects in Fluids. Cambridge, UK: Cambridge University Press. Wagner, W. 1998 Properties of Water and Steam. Springer-Verlag.

Zeren, R. W. \& Reynolds, W. C. 1972 Thermal instabilities in two-fluid horizontal layers. J. Fluid Mech. 53, 305-327.

\section{Appendix}

Here we consider the limits of large and small wavenumbers for the inert and twophase systems. We introduce the dimensionless layer widths $\bar{H}_{\alpha}=H_{\alpha} / d=1 /(1-\ell)$ and $\bar{H}_{\beta}=H_{\beta} / d=\ell /(1-\ell)$, where $d=H_{\alpha}+H_{\beta}$ and $\ell=-H_{\beta} / H_{\alpha}=-1 / 2$ in our calculations.

\subsection{Small Wavenumbers}

\subsubsection{Inert Case for General Ra}

By performing long-wavelength asymptotics for $\mathrm{Bo} \neq 0$ and $\mathrm{Cr} \neq 0$ we find the leading order result

$$
\mathrm{Ma}=\frac{c_{1} \operatorname{Bo}\left(\rho^{*}-1\right)}{\operatorname{Cr}\left(c_{2}+c_{5} \Gamma\right)}
$$

where

$$
\begin{gathered}
c_{1}=80 \bar{H}_{\alpha} \bar{H}_{\beta}\left[\bar{H}_{\alpha}+k^{*} \bar{H}_{\beta}\right]\left[\bar{H}_{\alpha}+\mu^{*} \bar{H}_{\beta}\right], \\
c_{2}=120\left[\bar{H}_{\alpha}^{2}-\mu^{*} \bar{H}_{\beta}^{2}\right] \\
c_{5}=\frac{2\left(1-k^{*}\right)}{k^{*}}\left\{11 \bar{H}_{\alpha}^{2} \bar{H}_{\beta}^{2}\left[\eta^{*} \mu^{*} \rho^{*} \bar{H}_{\alpha}-k^{*} \bar{H}_{\beta}\right]\right. \\
\left.+3\left[\eta^{*} \rho^{*} \bar{H}_{\alpha}^{5}-k^{*} \mu^{*} \bar{H}_{\beta}^{5}\right]+14 \bar{H}_{\alpha} \bar{H}_{\beta}\left[\eta^{*} \rho^{*} \bar{H}_{\alpha}^{3}-k^{*} \mu^{*} \bar{H}_{\beta}^{3}\right]\right\} .
\end{gathered}
$$


This expression is in good agreement with the numerical results for small-wavenumbers shown in Fig. 3, Fig. 5, Fig. 6, and Fig. 7. For Ra =0, this expression agrees with that given by Zeren \& Reynolds (1972) up to a difference in sign; their expression also agrees with their numerical results for $\mathrm{Ra}=0$ if the sign of their expression is changed. For $\mathrm{Ra} \neq 0$ our expression gives results which differ in both magnitude and sign from that given by Zeren \& Reynolds.

For the values given in Table II, we have

$$
c_{1}=12.8604, \quad c_{2}=46.5867, \quad c_{5}=1.1830
$$

\subsubsection{Inert Case for $\mathrm{Ra}=0$}

If we set $\mathrm{Ra}=0$ the linear eigenfunctions for the general problem can be written down explicitly, and the dispersion relation for $\sigma=0$ can be evaluated symbolically in closed form, though the resulting expression is very lengthy and difficult to interpret. However, the result can be expanded for small wavenumbers, and produces expressions that are not too unwieldy in special cases. Expressions for general values of the geometrical and materials parameters are given in the supplementary material.

The general expression for $\sigma=0$ and $\mathrm{Ra}=0$ in the inert case can be found by expanding in the wavenumber $a$ to give

$$
\begin{gathered}
0=a^{10}\left\{c_{1}\left[\rho^{*}-1\right] \mathrm{Bo}-c_{2} \mathrm{CrMa}\right\} \\
+a^{12}\left\{-\mathrm{Ma}\left(c_{3} \mathrm{Cr}+c_{4}\left[\rho^{*}-1\right] \mathrm{Bo}\right)-\left(c_{1}-c_{6}\left[\rho^{*}-1\right] \mathrm{Bo}\right)\right\}+O\left(a^{14}\right) .
\end{gathered}
$$

The expression can be solved for Ma to give a rational expression of the form

$$
\mathrm{Ma}=\frac{c_{1}\left[\rho^{*}-1\right] \mathrm{Bo}-\left(c_{1}-c_{6}\left[\rho^{*}-1\right] \mathrm{Bo}\right) a^{2}}{c_{2} \mathrm{Cr}+\left(c_{3} \mathrm{Cr}+c_{4}\left[\rho^{*}-1\right] \mathrm{Bo}\right) a^{2}}+O\left(a^{4}\right) .
$$

The constants $c_{1}$ and $c_{2}$ are as given above, and the additional constants $c_{3}, c_{4}$, and $c_{6}$ are given by

$$
\begin{gathered}
c_{3}=4\left[\bar{H}_{\alpha}+\bar{H}_{\beta}\right]\left(9\left[\bar{H}_{\alpha}^{4}-\mu^{*} \bar{H}_{\beta}^{4}\right]+10 \bar{H}_{\alpha} \bar{H}_{\beta}\left[\bar{H}_{\alpha}^{2}-\mu^{*} \bar{H}_{\beta}^{2}\right]+5 \bar{H}_{\alpha}^{2} \bar{H}_{\beta}^{2}\left[1-\mu^{*}\right]\right) \\
c_{4}=\frac{\bar{H}_{\alpha}^{3} \bar{H}_{\beta}^{3}}{\kappa^{*}}\left[\bar{H}_{\alpha}^{2}-\kappa^{*} \bar{H}_{\beta}^{2}\right] \\
c_{6}=\frac{8}{3} \bar{H}_{\alpha} \bar{H}_{\beta}\left(9\left[\bar{H}_{\alpha}^{4}+k^{*} \mu^{*} \bar{H}_{\beta}^{4}\right]+21 \bar{H}_{\alpha}^{2} \bar{H}_{\beta}^{2}\left[1+k^{*} \mu^{*}\right]\right. \\
\left.+19 \bar{H}_{\alpha} \bar{H}_{\beta}\left[k^{*} \bar{H}_{\alpha}^{2}+\mu^{*} \bar{H}_{\beta}^{2}\right]+11 \bar{H}_{\alpha} \bar{H}_{\beta}\left[k^{*} \bar{H}_{\beta}^{2}+\mu^{*} \bar{H}_{\alpha}^{2}\right]\right)
\end{gathered}
$$

We note that the dependence on the Bond number Bo enters through the quantity $\left[\rho^{*}-1\right] \mathrm{Bo}-a^{2}$. For the values given in Table II, the additional constants are given by

$$
c_{3}=10.825, \quad c_{4}=4.73587 \times 10^{-3}, \quad c_{6}=3.10159 .
$$

We next consider some special cases. If $\mathrm{Cr} \neq 0$ and $\mathrm{Bo} \neq 0$, then to leading order in $a$ we have

$$
-\mathrm{Ma}=\frac{c_{1}\left[1-\rho^{*}\right] \mathrm{Bo}}{c_{2} \mathrm{Cr}}
$$

If $\mathrm{Cr}=0$, the two-term expansion is

$$
\mathrm{Ma}=\frac{c_{1}}{c_{4}} a^{-2}+\frac{c_{6}}{c_{4}},
$$

which is independent of Bo. This is a result of the boundary condition (2.34); for $\mathrm{Cr}=0$, the interface is rigid and the Bond number has no effect on the system. 
If $\mathrm{Bo}=0$ and $\mathrm{Cr} \neq 0$, the leading order result is

$$
-\mathrm{Ma}=\frac{c_{1}}{c_{2} \mathrm{Cr}} a^{2} .
$$

\subsubsection{Two-Phase Case for General Ra}

Long-wavelength asymptotics can also be performed in the two-phase case for general values of Ra. Since there are several possible cases to consider for the two-phase system depending on the assumed values of $\mathrm{Ma}, \mathrm{Ra}, \mathcal{L}_{\alpha \beta}$, and $\mathcal{S}_{\alpha \beta}$, we use the dimensionless temperature gradient $\mathcal{G}$ as a control parameter, so that the dimensionless parameters $\widetilde{\mathrm{Ma}}$, $\widetilde{\operatorname{Ra}}, \widetilde{\mathcal{L}}_{\alpha \beta}$, and $\widetilde{\mathcal{S}}_{\alpha \beta}$ can all appear as independent quantities in the expansion coefficients. For $\mathrm{Bo} \neq 0$ and $\mathrm{Cr} \neq 0$ we find the leading order result

$$
\mathcal{G}=\frac{d_{1} \operatorname{Bo}\left(\rho^{*}-1\right)}{\operatorname{Cr}\left(d_{2}+d_{5} \widetilde{\mathrm{Ra}}\right)}
$$

where

$$
\begin{gathered}
d_{1}=\frac{4}{3 \rho^{*}}\left[\bar{H}_{\alpha}+k^{*} \bar{H}_{\beta}\right]\left\{4 \bar{H}_{\alpha} \bar{H}_{\beta} \mu^{*}\left[\bar{H}_{\beta}^{2}+\left(\rho^{*}\right)^{2} \bar{H}_{\alpha}^{2}\right]\right. \\
\left.+6 \bar{H}_{\alpha}^{2} \bar{H}_{\beta}^{2} \mu^{*} \rho^{*}+\left[\bar{H}_{\beta}^{4}\left(\mu^{*}\right)^{2}+\bar{H}_{\alpha}^{4}\left(\rho^{*}\right)^{2}\right]\right\} \\
d_{2}=d_{2}^{(1)} \widetilde{\mathrm{Ma}}+d_{2}^{(2)} \widetilde{\mathcal{S}}_{\alpha \beta}, \\
d_{2}^{(1)}=\frac{8}{\rho^{*}}\left[\bar{H}_{\alpha}+\bar{H}_{\beta}\right] \bar{H}_{\alpha} \bar{H}_{\beta} \mu^{*}\left[1-\rho^{*}\right]\left[\bar{H}_{\beta}+\rho^{*} \bar{H}_{\alpha}\right] \\
d_{2}^{(2)}=\frac{4}{3}\left[\bar{H}_{\alpha}+\bar{H}_{\beta}\right]\left(3 \bar{H}_{\alpha}^{2} \bar{H}_{\beta}^{2} \mu^{*}\left[1+\rho^{*}\right]\right. \\
\left.+4 \bar{H}_{\alpha} \bar{H}_{\beta} \mu^{*}\left[\bar{H}_{\beta}^{2}+\rho^{*} \bar{H}_{\alpha}^{2}\right]+\left[\bar{H}_{\alpha}^{4} \rho^{*}+\bar{H}_{\beta}^{4}\left(\mu^{*}\right)^{2}\right]\right) \\
d_{5}=\frac{\left(1-k^{*}\right)\left(1-\rho^{*}\right)}{15 k^{*} \rho^{*}}\left\{23 \mu^{*} H_{a} H_{b}\left[k^{*} H_{b}^{4}+\eta^{*}\left(\rho^{*}\right)^{2} H_{a}^{4}\right]\right. \\
\left.+7\left[k^{*}\left(\mu^{*}\right)^{2} H_{b}^{6}+\eta^{*}\left(\rho^{*}\right)^{2} H_{a}^{6}\right]+15 \mu^{*} \rho^{*} H_{a}^{2} H_{b}^{2}\left[k^{*} H_{b}^{2}+\eta^{*} H_{a}^{2}\right]\right\}
\end{gathered}
$$

For the values given in Table II, we have

$$
\begin{gathered}
d_{1}=0.508715, \quad d_{2}=2.36561 \times 10^{14}, \quad d_{5}=0.032388 \\
d_{2}^{(1)}=0.893976, \quad d_{2}^{(2)}=0.402588
\end{gathered}
$$

8.1.4. Two-Phase Case for $\widetilde{\mathrm{Ra}}=0$

For the two-phase case with $\widetilde{\mathrm{Ra}}=0$ the dispersion relation for $\sigma=0$ can again be evaluated symbolically in closed form. The result can then be expanded for small wavenumbers, giving

$$
\begin{gathered}
0=a^{8}\left\{d_{1}\left[\rho^{*}-1\right] \mathrm{Bo}-d_{2} \mathrm{Cr} \mathcal{G}\right\} \\
+a^{10}\left\{-\mathcal{G}\left(d_{3} \mathrm{Cr}+d_{4}\left[\rho^{*}-1\right] \mathrm{Bo}\right)-\left(d_{1}-d_{6}\left[\rho^{*}-1\right] \mathrm{Bo}\right)\right\}+O\left(a^{12}\right) .
\end{gathered}
$$

The expression can be solved for $\mathcal{G}$ as a rational expression of the form

$$
\mathcal{G}=\frac{d_{1}\left[\rho^{*}-1\right] \mathrm{Bo}-\left(d_{1}-d_{6}\left[\rho^{*}-1\right] \mathrm{Bo}\right) a^{2}}{d_{2} \mathrm{Cr}+\left(d_{3} \mathrm{Cr}+d_{4}\left[\rho^{*}-1\right] \mathrm{Bo}\right) a^{2}}+O\left(a^{4}\right) .
$$

This expression is similar in form to the corresponding expression (8.7) for the inert case. The various limiting cases therefore have the same form as for the inert case, though the 
coefficients $d_{j}$ and $c_{j}$ are quite different in magnitude and, for $d_{4}$ and $c_{4}$, differ in sign. The coefficients $d_{1}$ and $d_{2}$ are as given above, and $d_{3}, d_{4}$, and $d_{6}$ are given by

$$
\begin{aligned}
d_{3} & =\widetilde{\operatorname{Ma}} d_{3}^{(1)}+\widetilde{\mathcal{S}}_{\alpha \beta} d_{3}^{(2)} \\
d_{4} & =\widetilde{\operatorname{Ma}} d_{4}^{(1)}+\widetilde{\mathcal{S}}_{\alpha \beta} d_{4}^{(2)} \\
d_{6} & =d_{6}^{(1)}+\widetilde{\operatorname{Ma}} \widetilde{\mathcal{L}}_{\alpha \beta} d_{6}^{(2)}+\widetilde{\mathcal{L}}_{\alpha \beta} \widetilde{\mathcal{S}}_{\alpha \beta} d_{6}^{(3)},
\end{aligned}
$$

with

$$
\begin{aligned}
& d_{3}^{(1)}=\frac{4}{3 \rho^{*}}\left[\bar{H}_{\alpha}+\bar{H}_{\beta}\right] \bar{H}_{\alpha} \bar{H}_{\beta} \times \\
& \left(3 \bar{H}_{\alpha} \bar{H}_{\beta} \mu^{*}\left[\bar{H}_{\alpha}-\left(\rho^{*}\right)^{2} \bar{H}_{\beta}\right]+2 \bar{H}_{\alpha} \bar{H}_{\beta} \mu^{*}\left[\bar{H}_{\beta}-\left(\rho^{*}\right)^{2} \bar{H}_{\alpha}\right]\right. \\
& +\mu^{*}\left[\bar{H}_{\beta}^{3}-\left(\rho^{*}\right)^{2} \bar{H}_{\alpha}^{3}\right]+2\left[\left(\mu^{*}\right)^{2} \bar{H}_{\beta}^{3}-\left(\rho^{*}\right)^{2} \bar{H}_{\alpha}^{3}\right] \\
& \left.+3 \mu^{*} \rho^{*}\left[\bar{H}_{\alpha}^{3}-\bar{H}_{\beta}^{3}\right]+\bar{H}_{\alpha} \bar{H}_{\beta} \mu^{*} \rho^{*}\left[\bar{H}_{\beta}-\bar{H}_{\alpha}\right]\right) \\
& d_{3}^{(2)}=\frac{2}{45}\left[\bar{H}_{\alpha}+\bar{H}_{\beta}\right] \times \\
& \left(15 \bar{H}_{\alpha}^{2} \bar{H}_{\beta}^{2} \mu^{*}\left[\bar{H}_{\alpha}^{2}+\rho^{*} \bar{H}_{\beta}^{2}\right]+90 \bar{H}_{\alpha}^{3} \bar{H}_{\beta}^{3} \mu^{*}\left[1+\rho^{*}\right]+55 \bar{H}_{\alpha}^{2} \bar{H}_{\beta}^{2} \mu^{*}\left[\bar{H}_{\beta}^{2}+\rho^{*} \bar{H}_{\alpha}^{2}\right]\right. \\
& +44 \bar{H}_{\alpha} \bar{H}_{\beta} \mu^{*}\left[\bar{H}_{\beta}^{4}+\rho^{*} \bar{H}_{\alpha}^{4}\right]+65 \bar{H}_{\alpha}^{2} \bar{H}_{\beta}^{2}\left[\left(\mu^{*}\right)^{2} \bar{H}_{\beta}^{2}+\rho^{*} \bar{H}_{\alpha}^{2}\right] \\
& \left.+10 \bar{H}_{\alpha} \bar{H}_{\beta}\left[\left(\mu^{*}\right)^{2} \bar{H}_{\beta}^{4}+\rho^{*} \bar{H}_{\alpha}^{4}\right]+9\left[\left(\mu^{*}\right)^{2} \bar{H}_{\beta}^{6}+\rho^{*} \bar{H}_{\alpha}^{6}\right]\right) \\
& d_{4}^{(1)}=\frac{-1}{30 \rho^{*} \kappa^{*}} \bar{H}_{\alpha}^{2} \bar{H}_{\beta}^{2} \times \\
& \left\{5 \bar{H}_{\alpha}^{2} \bar{H}_{\beta}^{2}\left[\bar{H}_{\beta} \mu^{*}-\bar{H}_{\alpha}\left(\rho^{*}\right)^{2} \kappa^{*}\right]-7 \bar{H}_{\alpha} \bar{H}_{\beta} \rho^{*}\left[\bar{H}_{\alpha}^{3}-\bar{H}_{\beta}^{3} \mu^{*} \kappa^{*}\right]-2\left[\bar{H}_{\alpha}^{5}\left(\rho^{*}\right)^{2}-\bar{H}_{\beta}^{5} \mu^{*} \kappa^{*}\right]\right\} \\
& d_{4}^{(2)}=\frac{-1}{90 \kappa^{*}} \bar{H}_{\alpha}^{3} \bar{H}_{\beta}^{3} \times \\
& \left(14 \bar{H}_{\alpha} \bar{H}_{\beta}\left[\bar{H}_{\alpha}^{2}+\bar{H}_{\beta}^{2} \mu^{*} \rho^{*} \kappa^{*}\right]+11 \bar{H}_{\alpha}^{2} \bar{H}_{\beta}^{2}\left[\mu^{*}+\rho^{*} \kappa^{*}\right]+3\left[\bar{H}_{\alpha}^{4} \rho^{*}+\bar{H}_{\beta}^{4} \mu^{*} \kappa^{*}\right]\right) \\
& d_{6}^{(1)}=\frac{2}{45 \rho^{*}}\left(60 \mu^{*} \bar{H}_{\alpha}^{3} \bar{H}_{\beta}^{3}\left[\bar{H}_{\alpha}+k^{*}\left(\rho^{*}\right)^{2} \bar{H}_{\beta}\right]+84 \mu^{*} \bar{H}_{\alpha}^{2} \bar{H}_{\beta}^{2}\left[\bar{H}_{\beta}^{3}+k^{*}\left(\rho^{*}\right)^{2} \bar{H}_{\alpha}^{3}\right]\right. \\
& +100 \mu^{*} \bar{H}_{\alpha}^{3} \bar{H}_{\beta}^{3}\left[k^{*} \bar{H}_{\beta}+\left(\rho^{*}\right)^{2} \bar{H}_{\alpha}\right]+44 \mu^{*} \bar{H}_{\alpha} \bar{H}_{\beta}\left[k^{*} \bar{H}_{\beta}^{5}+\left(\rho^{*}\right)^{2} \bar{H}_{\alpha}^{5}\right] \\
& +35 \bar{H}_{\alpha}^{3} \bar{H}_{\beta}^{3}\left[\left(\mu^{*}\right)^{2} \bar{H}_{\beta}+k^{*}\left(\rho^{*}\right)^{2} \bar{H}_{\alpha}\right]+19 \bar{H}_{\alpha} \bar{H}_{\beta}\left[\left(\mu^{*}\right)^{2} \bar{H}_{\beta}^{5}+k^{*}\left(\rho^{*}\right)^{2} \bar{H}_{\alpha}^{5}\right] \\
& +45 \bar{H}_{\alpha}^{2} \bar{H}_{\beta}^{2}\left[k^{*}\left(\mu^{*}\right)^{2} \bar{H}_{\beta}^{3}+\left(\rho^{*}\right)^{2} \bar{H}_{\alpha}^{3}\right]+9\left[k^{*}\left(\mu^{*}\right)^{2} \bar{H}_{\beta}^{7}+\left(\rho^{*}\right)^{2} \bar{H}_{\alpha}^{7}\right] \\
& \left.+60 \mu^{*} \rho^{*} \bar{H}_{\alpha}^{2} \bar{H}_{\beta}^{2}\left[\bar{H}_{\alpha}^{3}+k^{*} \bar{H}_{\beta}^{3}\right]+120 \mu^{*} \rho^{*} \bar{H}_{\alpha}^{3} \bar{H}_{\beta}^{3}\left[\bar{H}_{\beta}+k^{*} \bar{H}_{\alpha}\right]\right) \text {. } \\
& d_{6}^{(2)}=\frac{2}{3} \bar{H}_{\alpha}^{3} \bar{H}_{\beta}^{3}\left[\mu^{*} \bar{H}_{\beta}^{2}-\rho^{*} \bar{H}_{\alpha}^{2}\right] \\
& d_{6}^{(3)}=\frac{4}{9} \bar{H}_{\alpha}^{4} \bar{H}_{\beta}^{4} \rho^{*}\left[\bar{H}_{\alpha}+\mu^{*} \bar{H}_{\beta}\right] .
\end{aligned}
$$

For the values given in Table II, the additional constants are given by

$$
d_{3}=7.17145 \times 10^{13}, \quad d_{4}=-1.84596 \times 10^{11}, \quad d_{6}=6.3844 \times 10^{9} .
$$

Essentially the same values for $d_{j}$ are obtained by setting $\widetilde{\mathrm{Ma}}=0$; thus the small wavenumber behavior of the system is insensitive to the Marangoni effect for the parameters in Table II. 


\subsection{Large Wavenumbers}

We next consider the limit of large wavenumbers for a stationary mode. For our system the numerical results suggest that buoyancy effects and interface deformation are unimportant in this limit, so we also consider the formal limit of small Crispation number, $\mathrm{Cr} \rightarrow 0$, along with $\mathrm{Ra}=0$. For $\mathrm{Ra}=0$, the governing equations for the velocity field are decoupled from the thermal field, which simplifies the analysis. For $\mathrm{Cr} \rightarrow 0$ the dimensionless form of the normal momentum balance,

$$
\operatorname{Cr}\left(\tilde{p}^{\alpha}-\tilde{p}^{\beta}\right)-\operatorname{Bo}\left(\rho^{*}-1\right) \tilde{h}+a^{2} \tilde{h}=2 \operatorname{Cr}\left(\mu^{*} \tilde{w}_{z}^{\alpha}-\tilde{w}_{z}^{\beta}\right),
$$

then reduces to

$$
\operatorname{Bo}\left(\rho^{*}-1\right) \tilde{h}-a^{2} \tilde{h}=0 .
$$

For Bo $\left(\rho^{*}-1\right)-a^{2} \neq 0$, we conclude that the interface deformation vanishes, $\tilde{h}=0$.

In the limit of large wavenumber the disturbances are concentrated near the interface and the effects of the outer boundaries are insignificant. The appropriate solution can then be computed using decay conditions in an unbounded domain as $z \rightarrow \pm \infty$. The vertical components of the velocity field are given by

$$
\hat{w}^{\alpha}(z)=A_{\alpha} e^{-a z}+B_{\alpha} a z e^{-a z}, \quad \hat{w}^{\beta}(z)=A_{\beta} e^{a z}+B_{\beta} a z e^{a z} .
$$

The temperature fields are given by

$$
\begin{gathered}
\hat{T}^{\alpha}(z)=\frac{-G^{*} A_{\alpha}}{2 a^{2} \kappa^{*}}[a z] e^{-a z}-\frac{G^{*} B_{\alpha}}{4 a^{2} \kappa^{*}}[a z] e^{-a z}-\frac{G^{*} B_{\alpha}}{4 a^{2} \kappa^{*}}\left[a^{2} z^{2}\right] e^{-a z}+C_{\alpha} e^{-a z}, \\
\hat{T}^{\beta}(z)=\frac{A_{\beta}}{2 a^{2}}[a z] e^{a z}-\frac{B_{\beta}}{4 a^{2}}[a z] e^{a z}+\frac{B_{\beta}}{4 a^{2}}\left[a^{2} z^{2}\right] e^{a z}+C_{\beta} e^{a z} .
\end{gathered}
$$

The corresponding horizontal velocities and pressures are

$$
\tilde{u}^{\alpha}=i\left(B_{\alpha}-A_{\alpha}\right) e^{-a z}-i B_{\alpha} a z e^{-a z}, \quad \tilde{u}^{\beta}=i\left(B_{\beta}+A_{\beta}\right) e^{a z}+i B_{\beta} a z e^{a z},
$$

and

$$
\tilde{p}^{\alpha}=2 \rho^{*} \nu^{*} a B_{\alpha} e^{-a z}, \quad \tilde{p}^{\beta}=2 a B_{\beta} e^{a z} .
$$

These solutions are valid for both the inert and the two-phase problem, and the respective sets of interface boundary conditions determine the remaining six constants $A_{\alpha}, B_{\alpha}, C_{\alpha}$, $A_{\beta}, B_{\beta}$, and $C_{\beta}$ in each case.

\subsubsection{Inert Problem}

For the inert case with $\mathrm{Cr}=0$ the interfacial boundary conditions are

$$
\begin{gathered}
\tilde{w}^{\alpha}=0, \quad \tilde{w}^{\beta}=0, \quad \tilde{u}^{\alpha}=\tilde{u}^{\beta}, \\
\tilde{T}^{\alpha}=\tilde{T}^{\beta}, \quad k^{*} \tilde{T}_{z}^{\alpha}-\tilde{T}_{z}^{\beta}=0, \\
\left(\mu^{*} \tilde{u}_{z}^{\alpha}-\tilde{u}_{z}^{\beta}\right)+i a\left(\mu^{*} \tilde{w}^{\alpha}-\tilde{w}^{\beta}\right)-i a \operatorname{Ma} \tilde{T}^{\alpha}=0 .
\end{gathered}
$$

The velocity boundary conditions require $A_{\alpha}=A_{\beta}=0$ and $B_{\alpha}=B_{\beta}$, and the solution to the thermal problem then gives

$$
C_{\alpha}=C_{\beta}=-\left[\frac{\left(1-\kappa^{*}\right)}{4 a^{2} \kappa^{*}\left(1+k^{*}\right)}\right] B_{\alpha} .
$$

The remaining boundary condition give the dispersion relation,

$$
\mathrm{Ma}=8 a^{2}\left(1+\mu^{*}\right) \kappa^{*} \frac{\left(1+k^{*}\right)}{\left(1-\kappa^{*}\right)} .
$$


For the values in Table II this gives $\mathrm{Ma}=87.1 a^{2}$.

\subsubsection{Two-Phase Problem}

The boundary conditions for the two-phase case lead to the relations

$$
A_{\beta}=\rho^{*} A_{\alpha}, \quad B_{\beta}=B_{\alpha}-\left(1+\rho^{*}\right) A_{\alpha}, \quad C_{\beta}=C_{\alpha},
$$

and

$$
\begin{gathered}
A_{\alpha}=\frac{\operatorname{MaS}_{\alpha \beta}\left(1-\kappa^{*}\right)+8 a^{3} \kappa^{*}\left(\nu^{*}-1\right)\left(1+k^{*}\right)}{\Delta} C_{\alpha}, \\
B_{\alpha}=\frac{\operatorname{MaS}_{\alpha \beta} R-8 a^{3} \kappa^{*}\left(1+\rho^{*}\right)\left(1+k^{*}\right)}{\Delta} C_{\alpha}
\end{gathered}
$$

where

$$
\begin{gathered}
\Delta=2 a\left[\left(1+\rho^{*}\right)\left(1-\kappa^{*}\right)+R\left(\nu^{*}-1\right)\right], \\
R=-\left[\kappa^{*}\left(1+3 \rho^{*}\right)+2+4 a \kappa^{*} \rho^{*} \mathcal{L}_{\alpha \beta} / \mathrm{Ma}\right] .
\end{gathered}
$$

The dispersion relation takes the form

$$
\begin{gathered}
\left\{\frac{\Delta_{1}}{2\left(1+\mu^{*}\right)}-\mathcal{S}_{\alpha \beta}\left(1-\kappa^{*}-R_{1}\right)\right\} \mathrm{Ma} \\
=8 a^{3} \kappa^{*}\left(1+k^{*}\right)\left[\left(\nu^{*}+\rho^{*}\right)\right]-\mathcal{S}_{\alpha \beta} R_{2}-\frac{\Delta_{2}}{2\left(1+\mu^{*}\right)},
\end{gathered}
$$

where

$$
\begin{gathered}
R_{1}=-\left[\kappa^{*}\left(1+3 \rho^{*}\right)+2\right], \quad R_{2}=-4 a \kappa^{*} \rho^{*} \mathcal{L}_{\alpha \beta}, \\
\Delta_{1}=2 a\left[\left(1+\rho^{*}\right)\left(1-\kappa^{*}\right)+R_{1}\left(\nu^{*}-1\right)\right], \quad \Delta_{2}=2 a R_{2}\left(\nu^{*}-1\right) .
\end{gathered}
$$

For interpreting the "anomalous" two-phase mode, we consider the limit of large entropy $\mathcal{S}_{\alpha \beta}$, which produces some simplification by eliminating the Marangoni effect and making the interface isothermal with $\tilde{T}^{\alpha}=\tilde{T}^{\beta}=0$. We then have $C_{\alpha}=C_{\beta}=0$, $A_{\beta}=\rho^{*} A_{\alpha}, B_{\alpha}=A_{\alpha}$, and $B_{\beta}=-\rho^{*} A_{\alpha}$. We note that the steady-state vertical velocity fields and temperature fields then takes the form

$$
\begin{gathered}
w^{\alpha}(x, z)=A_{\alpha}[1+(a z)] e^{-a z} \cos a z, \quad w^{\beta}(x, z)=\rho^{*} A_{\alpha}[1-(a z)] e^{a z} \cos a z \\
T^{\alpha}(x, z)=G^{*} z-\frac{(a z) G^{*}}{4 a^{2} \kappa^{*}}[3+(a z)] A_{\alpha} e^{-a z} \cos a z \\
T^{\beta}(x, z)=z+\frac{(a z) \rho^{*}}{4 a^{2}}[3-(a z)] A_{\alpha} e^{a z} \cos a z .
\end{gathered}
$$

The resulting dispersion relation then has the simple form

$$
\frac{\mathrm{Ma}}{\mathcal{L}_{\alpha \beta}}=\frac{k_{\beta} G_{\beta}}{\rho_{\beta} L_{\alpha \beta}\left(\kappa_{\beta} / d\right)}=-\frac{4 \kappa^{*} \rho^{*}}{3\left(1+\kappa^{*} \rho^{*}\right)} a .
$$

This work was performed under the auspices of the U.S. Department of Energy by University of California, Lawrence Livermore National Laboratory under Contract W-7405-Eng-48. 
Table I

Thermophysical Properties of the Steam ( $\alpha$ phase) Water ( $\beta$ phase) system at the equilibrium state with $T_{E}=640 \mathrm{~K}$ and $p_{E}=202.7$ bar used in the numerical calculations $\dagger$

\begin{tabular}{lccc}
\hline \hline density of water & $\rho_{\beta}$ & 481.6 & $\mathrm{~kg} / \mathrm{m}^{3}$ \\
density of steam & $\rho_{\alpha}$ & 177.4 & $\mathrm{~kg} / \mathrm{m}^{3}$ \\
dynamic viscosity of water & $\mu_{\beta}$ & $5.526\left(10^{-5}\right)$ & $\mathrm{Pa} \mathrm{s}$ \\
dynamic viscosity of steam & $\mu_{\alpha}$ & $2.795\left(10^{-5}\right)$ & $\mathrm{Pa} \mathrm{s}$ \\
thermal conductivity of water & $k_{\beta}$ & 0.4177 & $\mathrm{~W} / \mathrm{mK}$ \\
thermal conductivity of steam & $k_{\alpha}$ & 0.2499 & $\mathrm{~W} / \mathrm{mK}$ \\
thermal diffusivity of water & $\kappa_{\beta}$ & $3.276\left(10^{-8}\right)$ & $\mathrm{m}^{2} / \mathrm{s}$ \\
thermal diffusivity of steam & $\kappa_{\alpha}$ & $2.682\left(10^{-8}\right)$ & $\mathrm{m}^{2} / \mathrm{s}$ \\
surface energy & $\gamma$ & $8.09\left(10^{-4}\right)$ & $\mathrm{J} / \mathrm{m}^{2}$ \\
$d \gamma / d T$ & $\gamma_{T}$ & $-1.42\left(10^{-4}\right)$ & $\mathrm{J} / \mathrm{K} \mathrm{m}^{2}$ \\
thermal expansion coefficient of water & $\eta_{\beta}$ & $3.94\left(10^{-2}\right)$ & $\mathrm{K}^{-1}$ \\
thermal expansion coefficient of steam & $\eta_{\alpha}$ & $7.34\left(10^{-2}\right)$ & $\mathrm{K}^{-1}$ \\
difference in enthalpy density (latent heat) & $L_{\alpha \beta}=h_{m}^{\alpha}-h_{m}^{\beta}$ & $5.524\left(10^{5}\right)$ & $\mathrm{J} / \mathrm{kg}$ \\
difference in entropy density & $s_{\alpha \beta}=s_{m}^{\alpha}-s_{m}^{\beta}$ & $8.632\left(10^{2}\right)$ & $\mathrm{J} / \mathrm{K} \mathrm{kg}$ \\
total thickness of layer & $d$ & $2.0\left(10^{-3}\right)$ & $\mathrm{m}$ \\
gravitational acceleration & $g$ & 9.8 & $\mathrm{~m} / \mathrm{s}^{2}$ \\
\hline
\end{tabular}

$\dagger$ The surface energy is given by $\gamma=235.8(1-\theta)^{1.256}[1-0.625(1-\theta)] \mathrm{mN} \mathrm{m}^{-1}$, where $\theta=T / T_{c}$ is the reduced temperature, and $T_{c}=647.096 \mathrm{~K}$ is the critical temperature (Parry et al. 2000; Wagner 1998). 


\section{Table II}

Dimensionless Variables for the Steam ( $\alpha$ phase) Water ( $\beta$ phase) system at $T_{E}=640$ $\mathrm{K}$ and $p_{E}=202.7$ bar

\begin{tabular}{lcl}
\hline \hline ratio of densities & $\rho^{*}$ & 0.368 \\
ratio of dynamic viscosities & $\mu^{*}$ & 0.506 \\
ratio of thermal conductivities & $k^{*}$ & 0.598 \\
ratio of thermal diffusivities & $\kappa^{*}$ & 0.819 \\
ratio of thermal expansion coefficients & $\eta^{*}$ & 1.86 \\
Marangoni number & $\widetilde{\mathrm{Ma}}$ & $1.004\left(10^{8}\right)$ \\
Rayleigh number & $\widetilde{\mathrm{Ra}}$ & $5.26\left(10^{8}\right)$ \\
dimensionless latent heat & $\widetilde{\mathcal{L}}_{\alpha \beta}$ & $3.27\left(10^{-2}\right)$ \\
dimensionless entropy difference & $\widetilde{\mathcal{S}}_{\alpha \beta}$ & $5.86\left(10^{14}\right)$ \\
Crispation number & $\mathrm{Cr}$ & $1.12\left(10^{-6}\right)$ \\
Bond number & $\mathrm{Bo}$ & 23.3 \\
Prandtl number & $\operatorname{Pr}$ & 3.50 \\
ratio of Marangoni and Rayleigh numbers & $\Gamma$ & 5.24 \\
thickness ratio & $\ell$ & $-1 / 2$ \\
& & \\
\hline
\end{tabular}




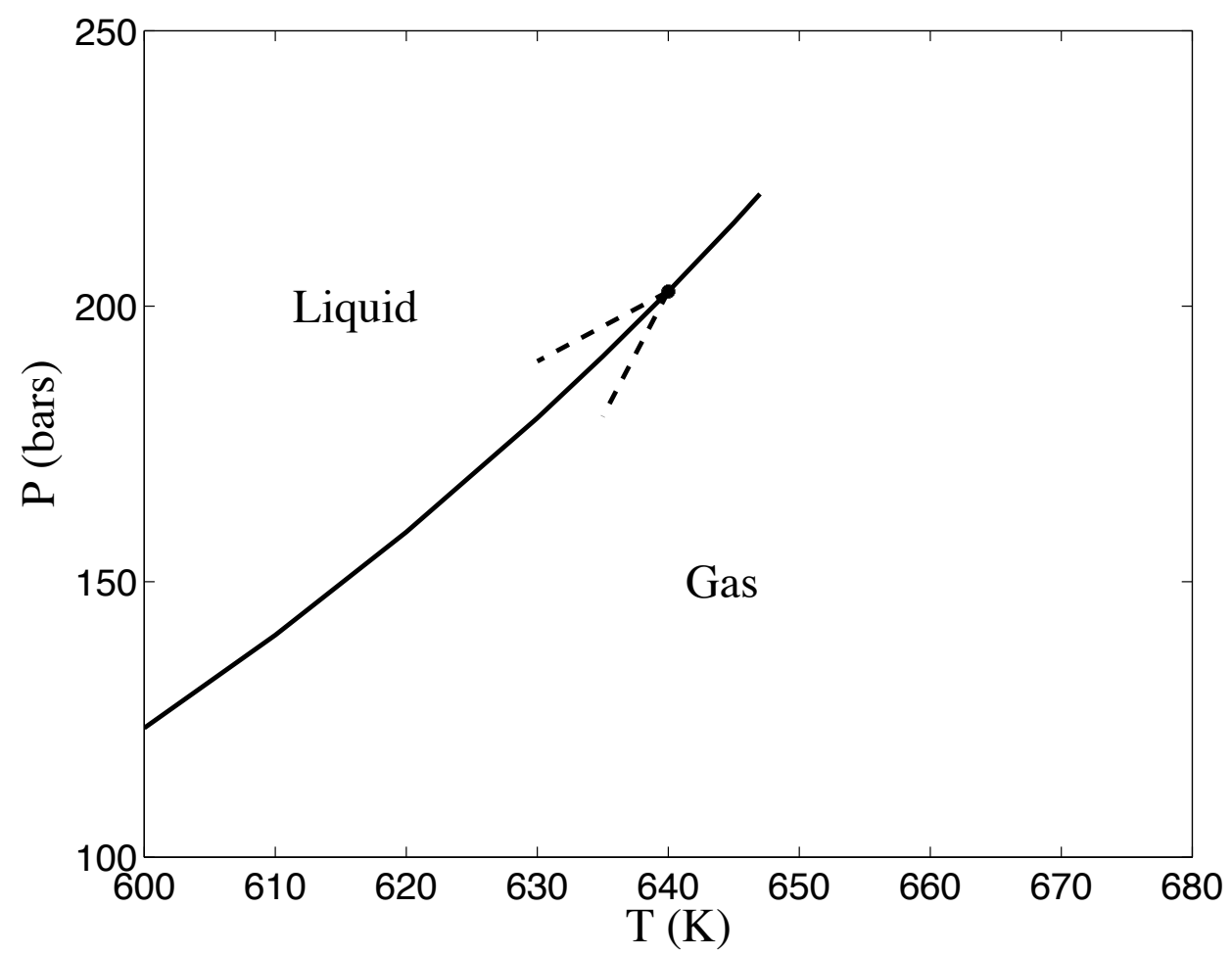

Figure 1. Phase diagram for the water-steam system near its critical point. The solid curve is the co-existence curve for the two phase system, representing the locus of equilibrium temperatures and pressures and terminating at the critical point where the properties of the liquid and gas phases become identical. The two dashed curves each represent a schematic profile of $T(z)$ and $P(z)$ in the gas layer. If the dashed curve has a small enough slope it lies within in the liquid region of the phase diagram and represents a supercooled gas state. A similar diagram applies for the profile in the liquid layer, where a superheated liquid state is possible if the corresponding slope is sufficiently small. 


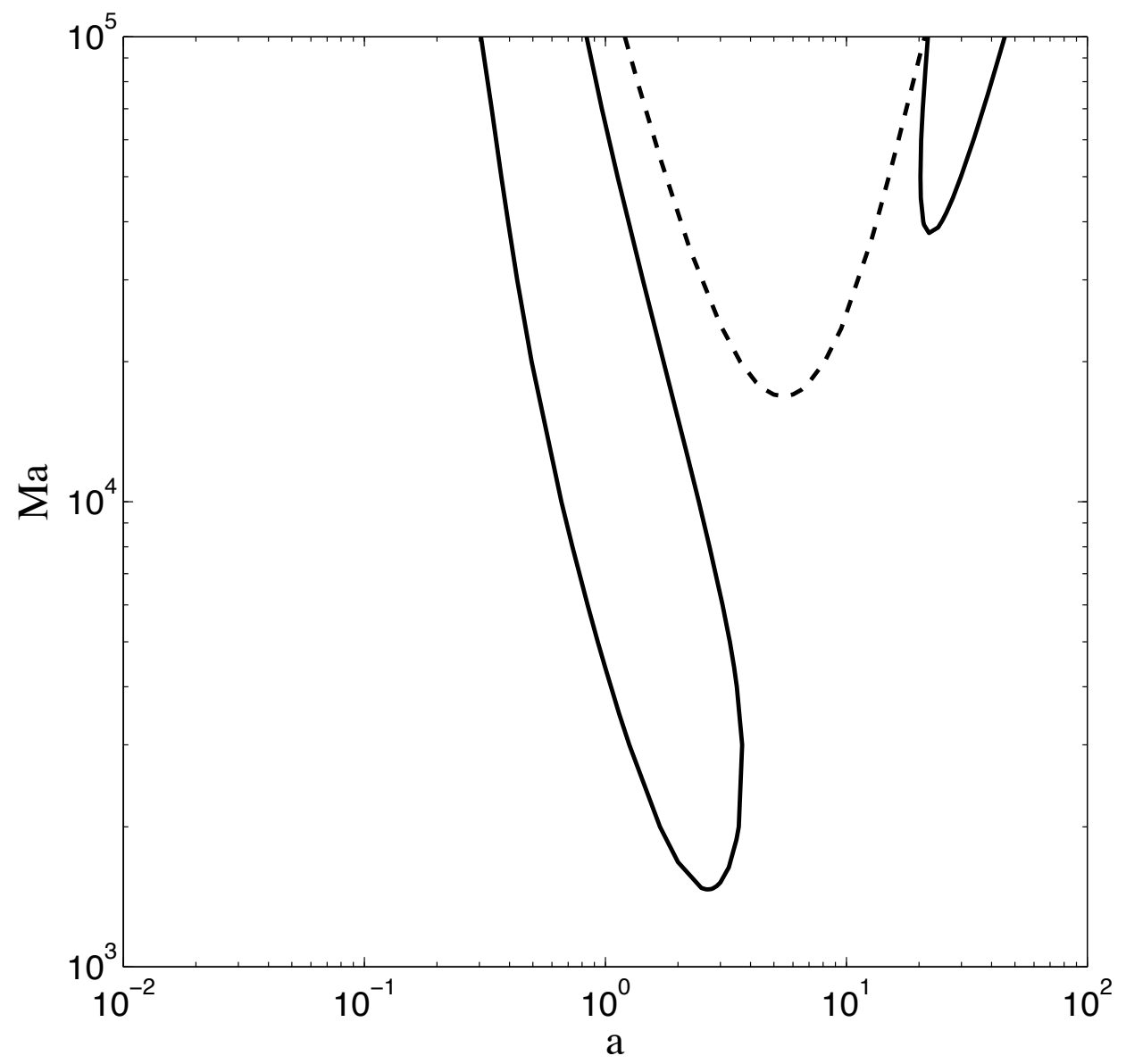

FiguRE 2. Marginal stability curves of Marangoni number Ma versus wavenumber $a$ for a system of benzene over water with heating from above. Solid curves represent stationary modes with $\sigma_{i}=0$, and the dashed curve is an oscillatory mode with $\sigma_{i} \neq 0$. 


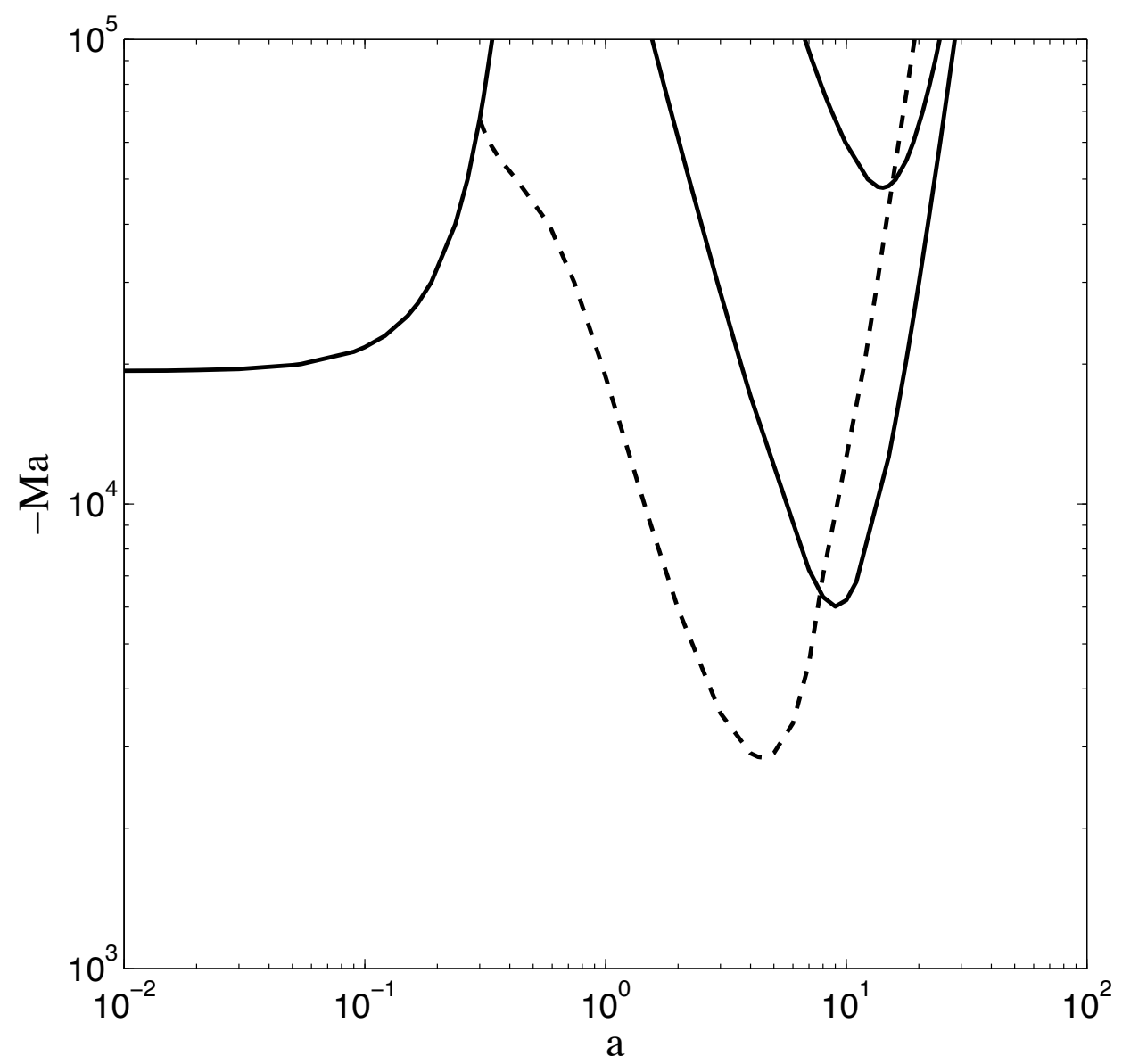

Figure 3. Marginal stability curves for a system of benzene over water with heating from below. Solid curves represent stationary modes with $\sigma_{i}=0$, and the dashed curve is an oscillatory mode with $\sigma_{i} \neq 0$. 


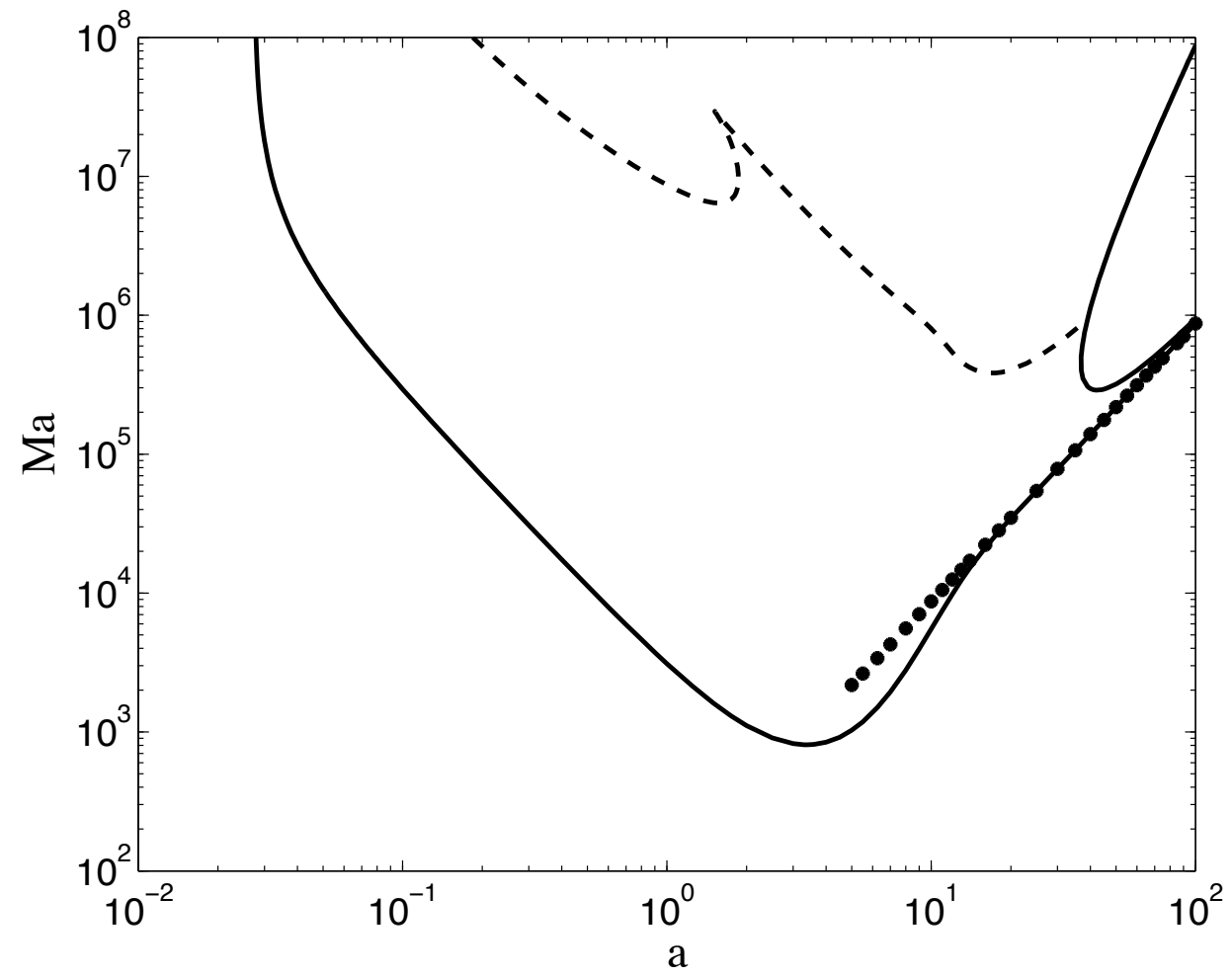

FiguRE 4. Marginal stability curves for an inert water-steam system heated from above. The lowest solid curve represents a stationary mode in which buoyancy effects have been eliminated by setting $\Gamma=0$. The upper solid curve and upper dashed curve include the effects of buoyancy, and correspond to stationary and oscillatory modes, respectively. The solid points correspond to a large-wavenumber approximation. 


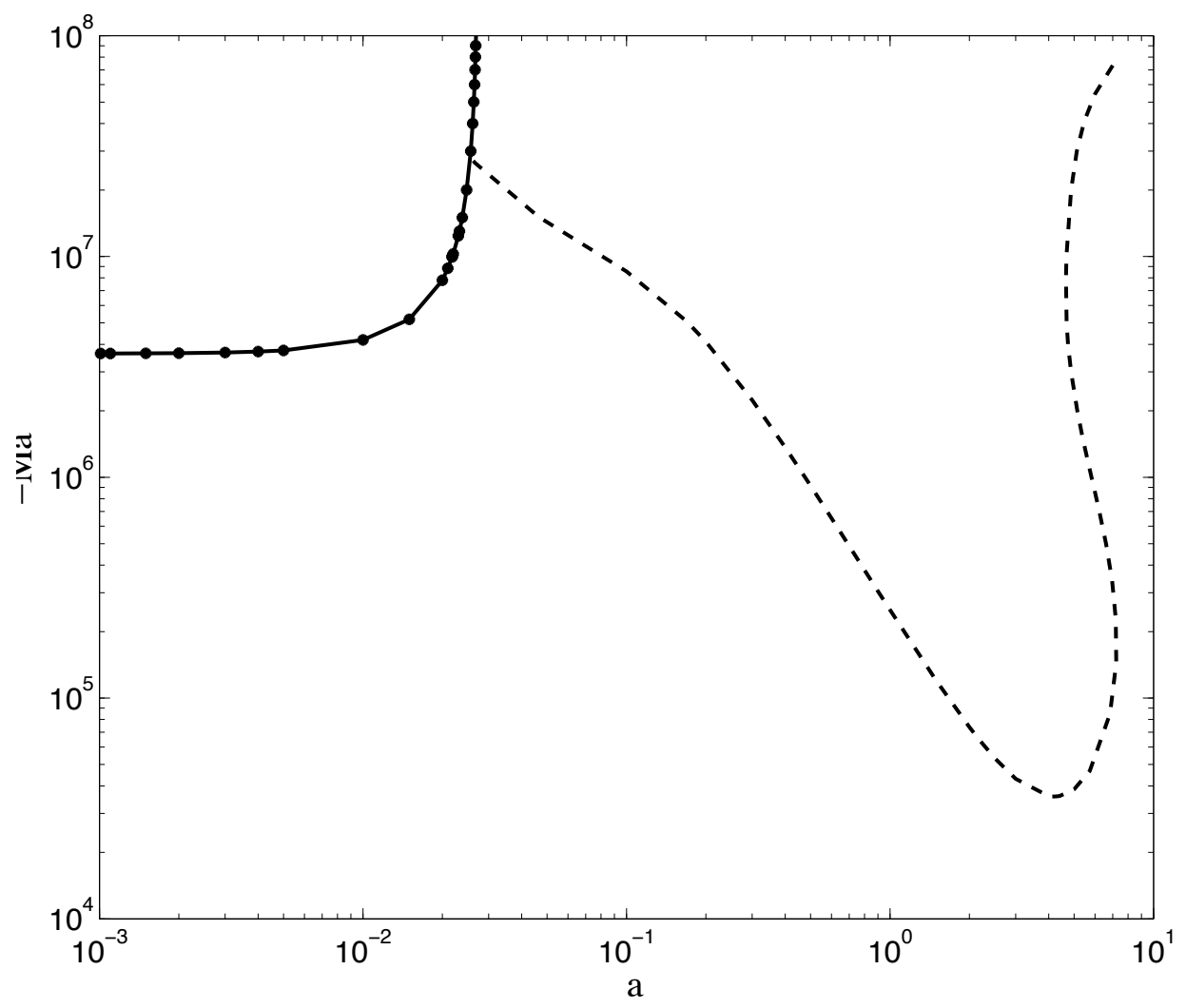

FiguRE 5. Marginal stability curves for an inert water-steam system heated from below. Buoyancy effects have been eliminated by setting $\Gamma=0$. The solid curve represents a stationary mode, and the dashed curve represents an oscillatory mode. The solid points correspond to a small-wavenumber approximation. 


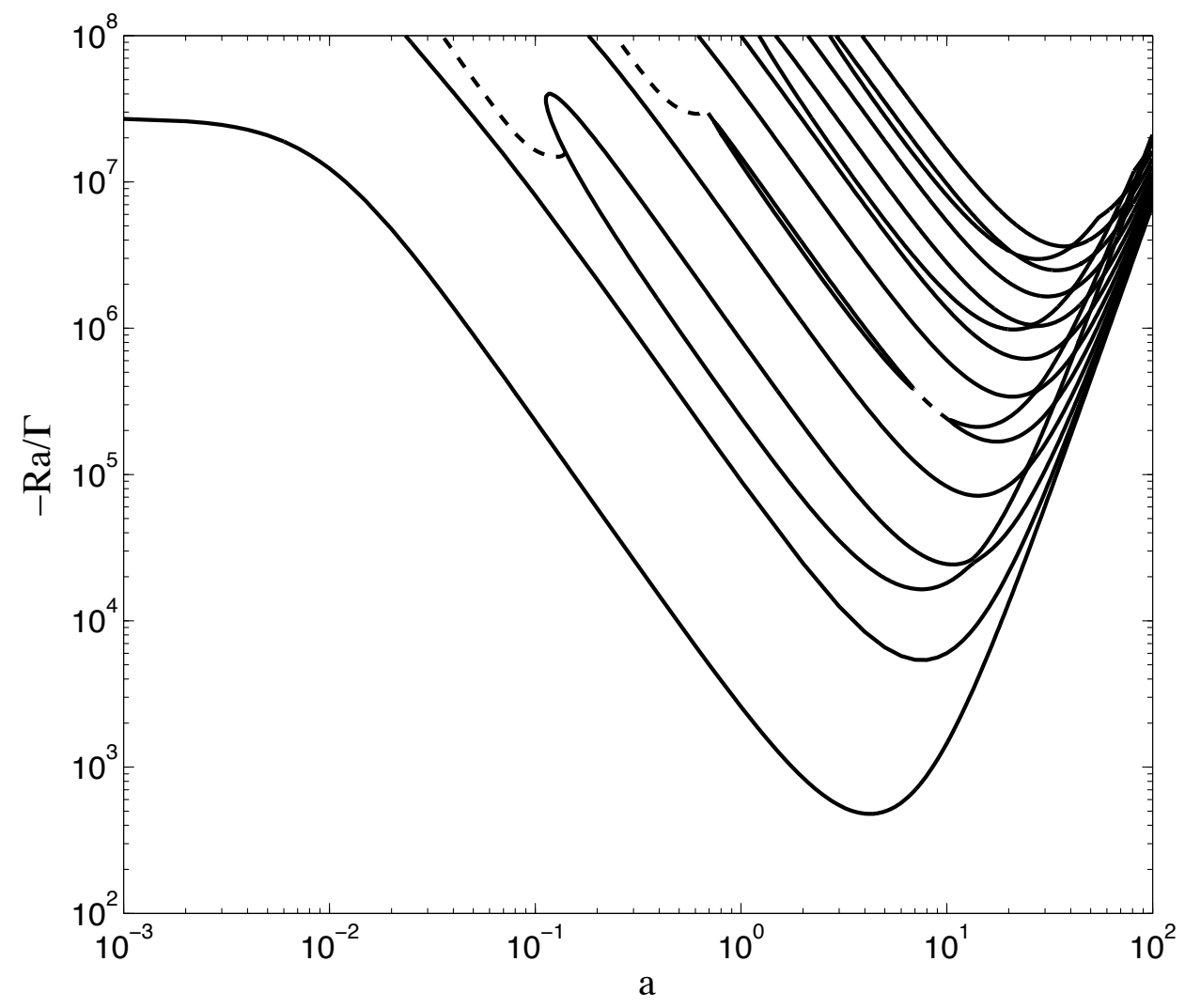

FiguRE 6. Marginal stability curves for an inert water-steam system heated from below. Marangoni effects have been eliminated by setting $\widetilde{\mathrm{Ma}}=0$. Solid curves represent stationary modes, and the dashed curves represent oscillatory modes. Here we plot the quantity $\mathrm{Ra} / \Gamma$ for $\Gamma=5.24$, so that the vertical axis scale is equal to that used in the previous figure. 


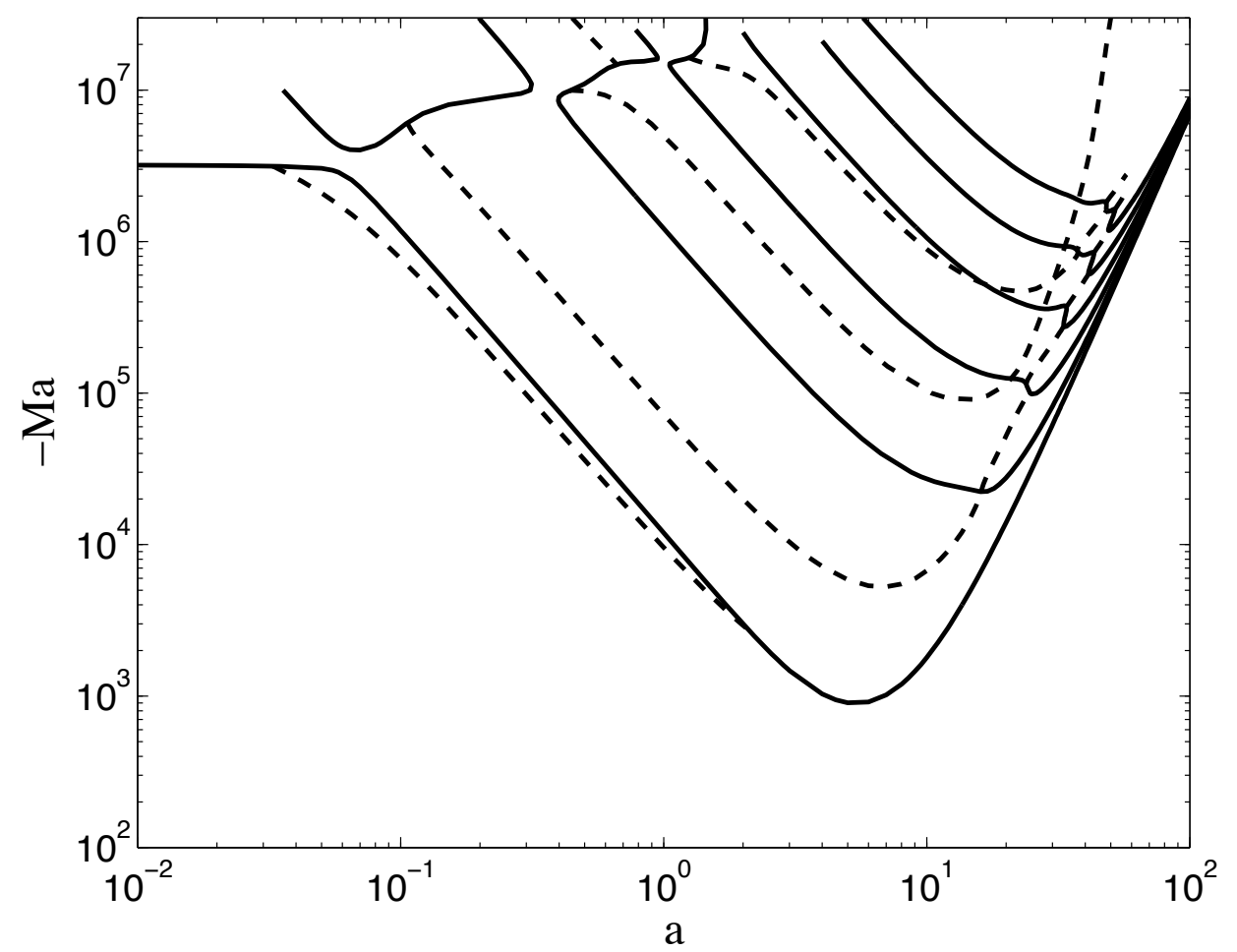

FiguRE 7. Marginal stability curves for an inert water-steam system heated from below. Both buoyancy and Marangoni effects are present. Solid curves represent stationary modes, and the dashed curves represent oscillatory modes. 


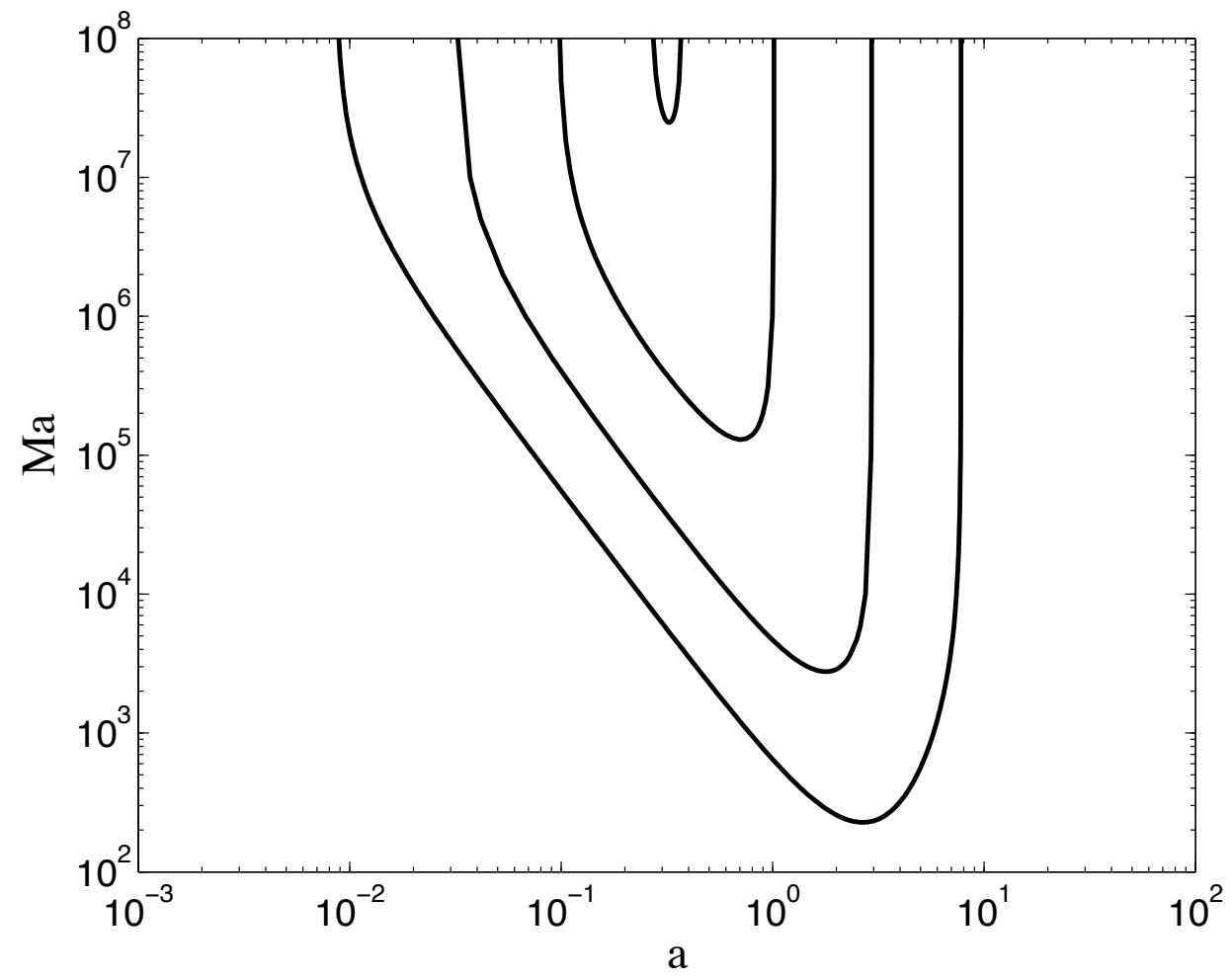

Figure 8. Marginal stability curves for a two-phase water-steam system heated from above for various values of the entropy jump $\mathcal{S}_{\alpha \beta}$, keeping the ratio $\mathcal{S}_{\alpha \beta} / \mathcal{L}_{\alpha \beta}$ fixed. Here the effects of buoyancy are neglected by setting $\Gamma=0$. From bottom to top the curves correspond to $\widetilde{\mathcal{S}}_{\alpha \beta}=0.0,2.5 \times 10^{8}, 2.9 \times 10^{8}$, and $2.955 \times 10^{8}$, respectively. 


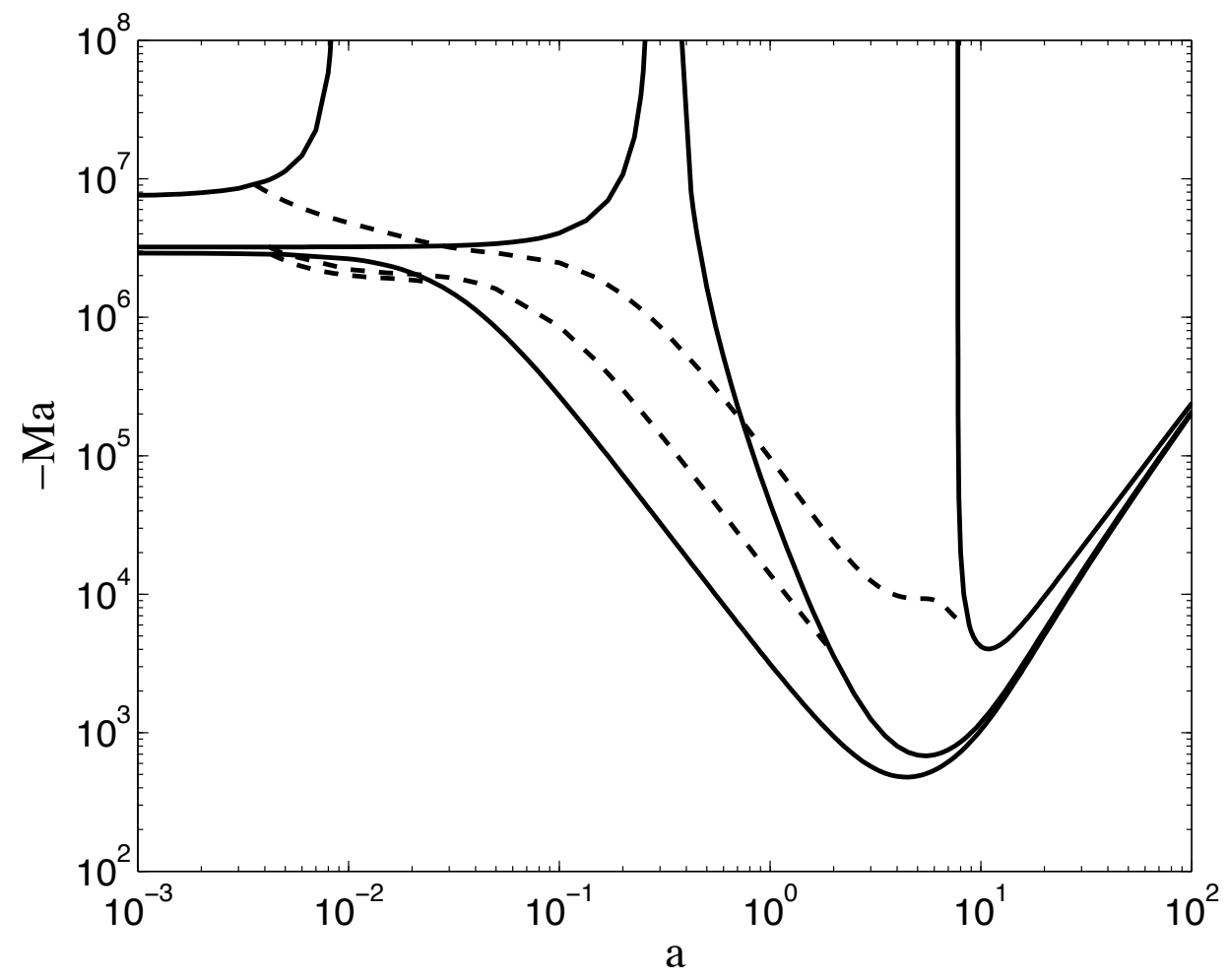

FIGURE 9. Marginal stability curves for a two-phase water-steam system heated from below for various values of the entropy jump $\mathcal{S}_{\alpha \beta}$, keeping the ratio $\mathcal{S}_{\alpha \beta} / \mathcal{L}_{\alpha \beta}$ fixed. Here the effects of buoyancy are neglected by setting $\Gamma=0$. The solid curves represent stationary modes, and dashed curves correspond to oscillatory modes that connect to stationary modes with the same values of $\mathcal{S}_{\alpha \beta}$. From top to bottom on either the extreme left or extreme right sides of the plot, the stationary curves correspond to $\widetilde{\mathcal{S}}_{\alpha \beta}=0,2.955 \times 10^{8}$, and $3.5 \times 10^{8}$, respectively. 


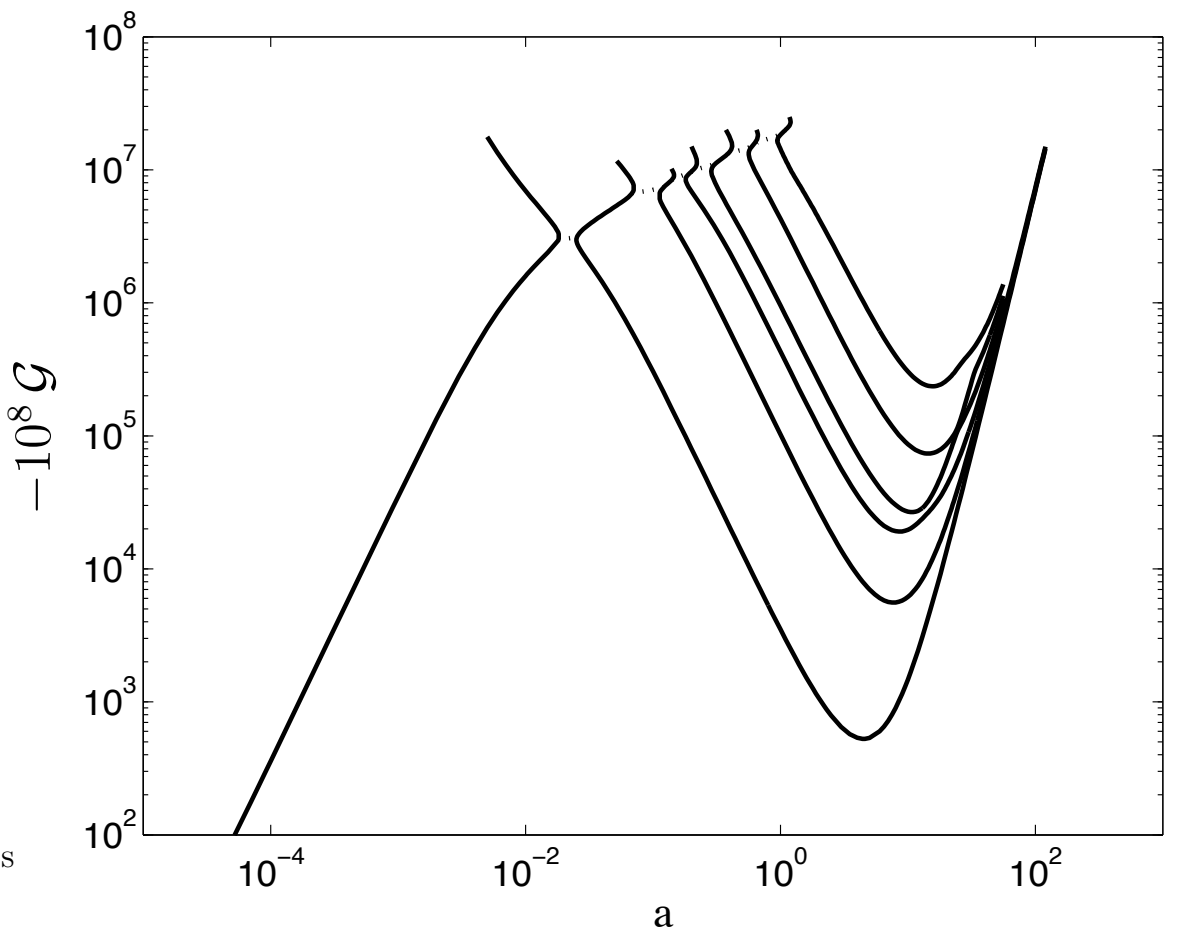

FiguRE 10. Marginal stability curves for a two-phase water-steam system heated from below. Solid curves represent stationary modes, and the dotted curves represent oscillatory modes. 


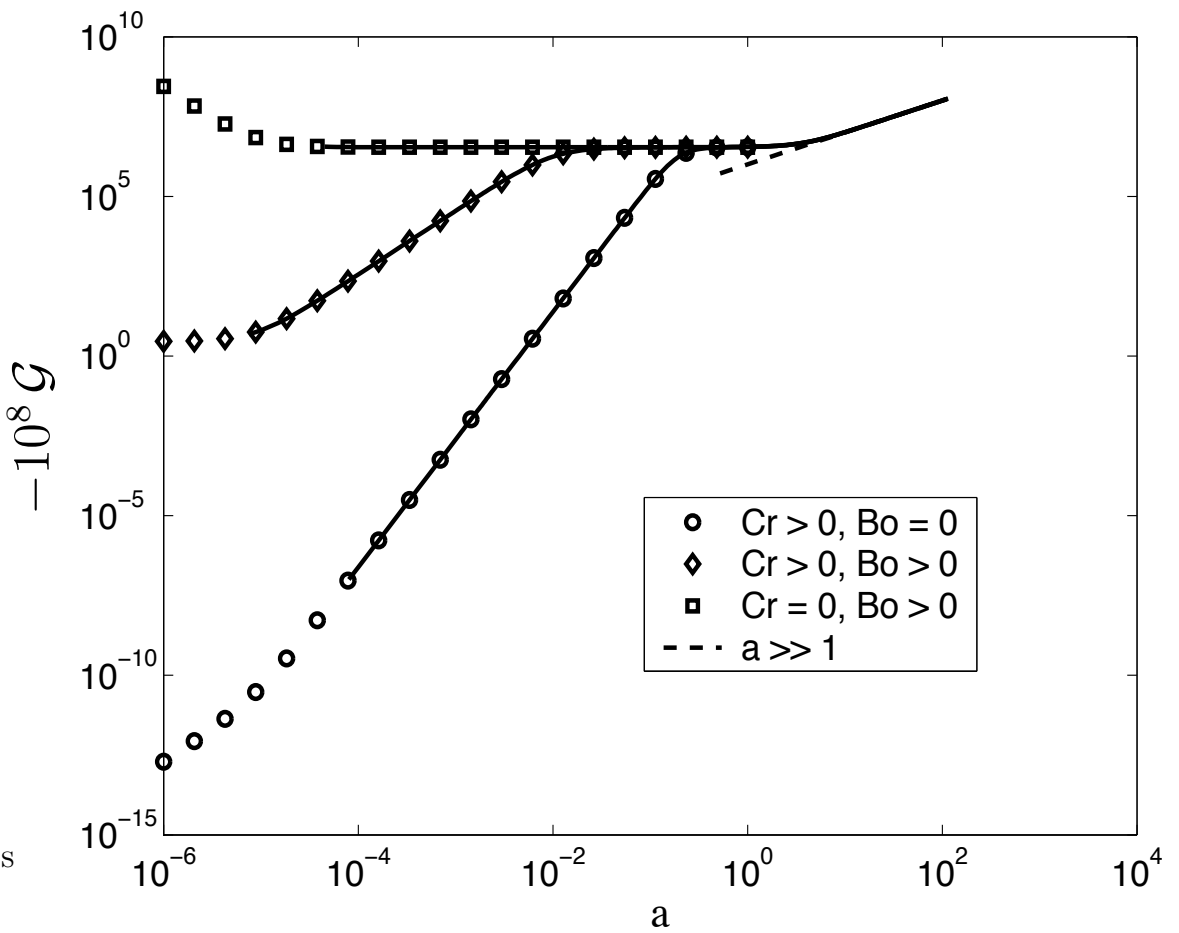

FIGURE 11. Marginal stability curves for a two-phase water-steam system heated from below. Here the effects of buoyancy are neglected by setting $\Gamma=0$. The solid curves represent numerical results, and the symbols correspond to a small wavenumber expansion. The dashed curve represents a large wavenumber expansion. 


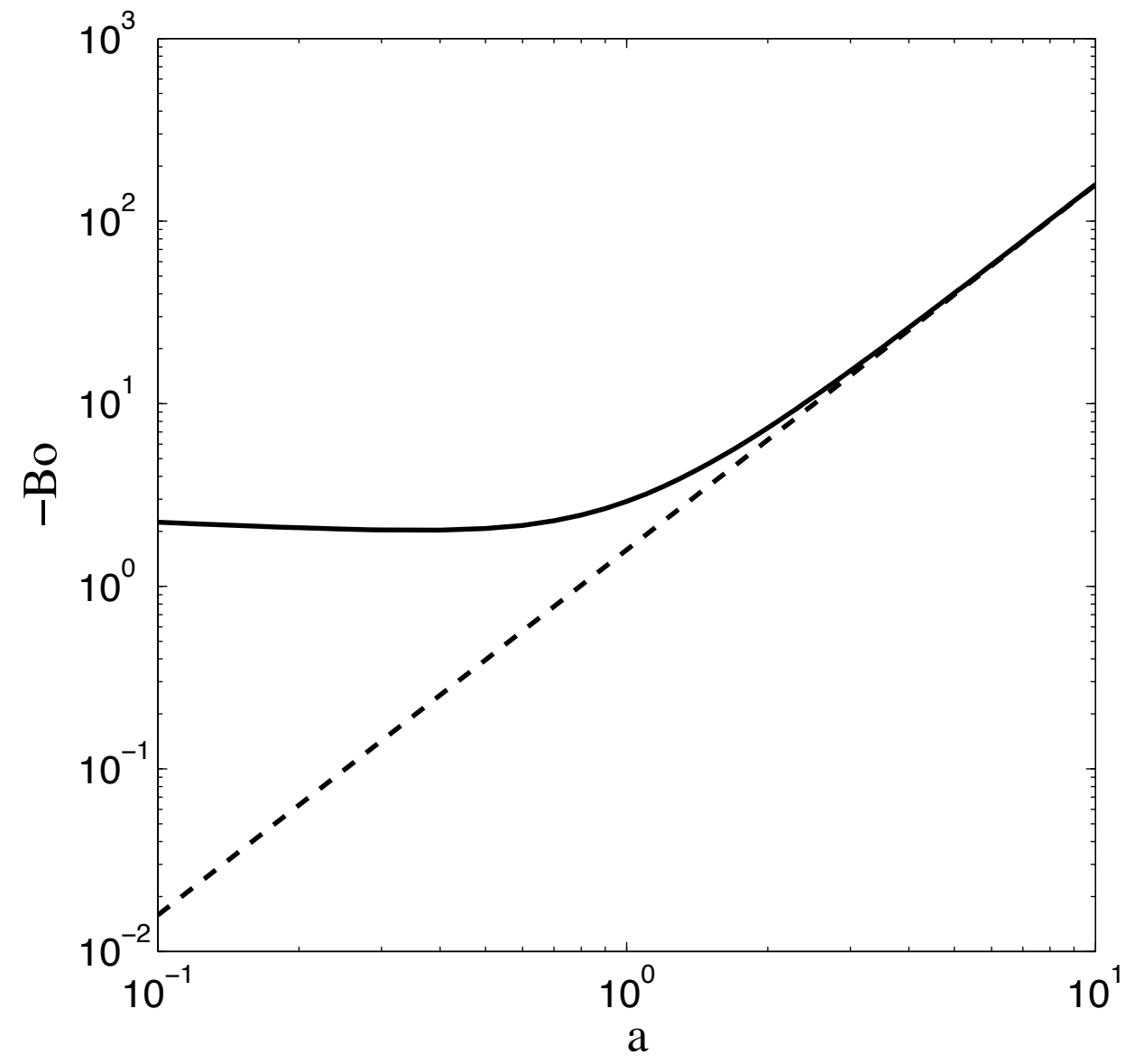

FIGURE 12. Marginal stability curves for a two-phase water-steam system that is unstably stratified with respect to gravity. The dashed curve corresponds to the classical Rayleigh-Taylor instability in the absence of buoyancy, given by $-\mathrm{Bo}=a^{2} /\left(1-\rho^{*}\right)$. The solid curve represents numerical results that include the effects of buoyancy, with $\mathrm{Ma}=-1.0 \times 10^{6}$ and $\Gamma=-5.24$. 


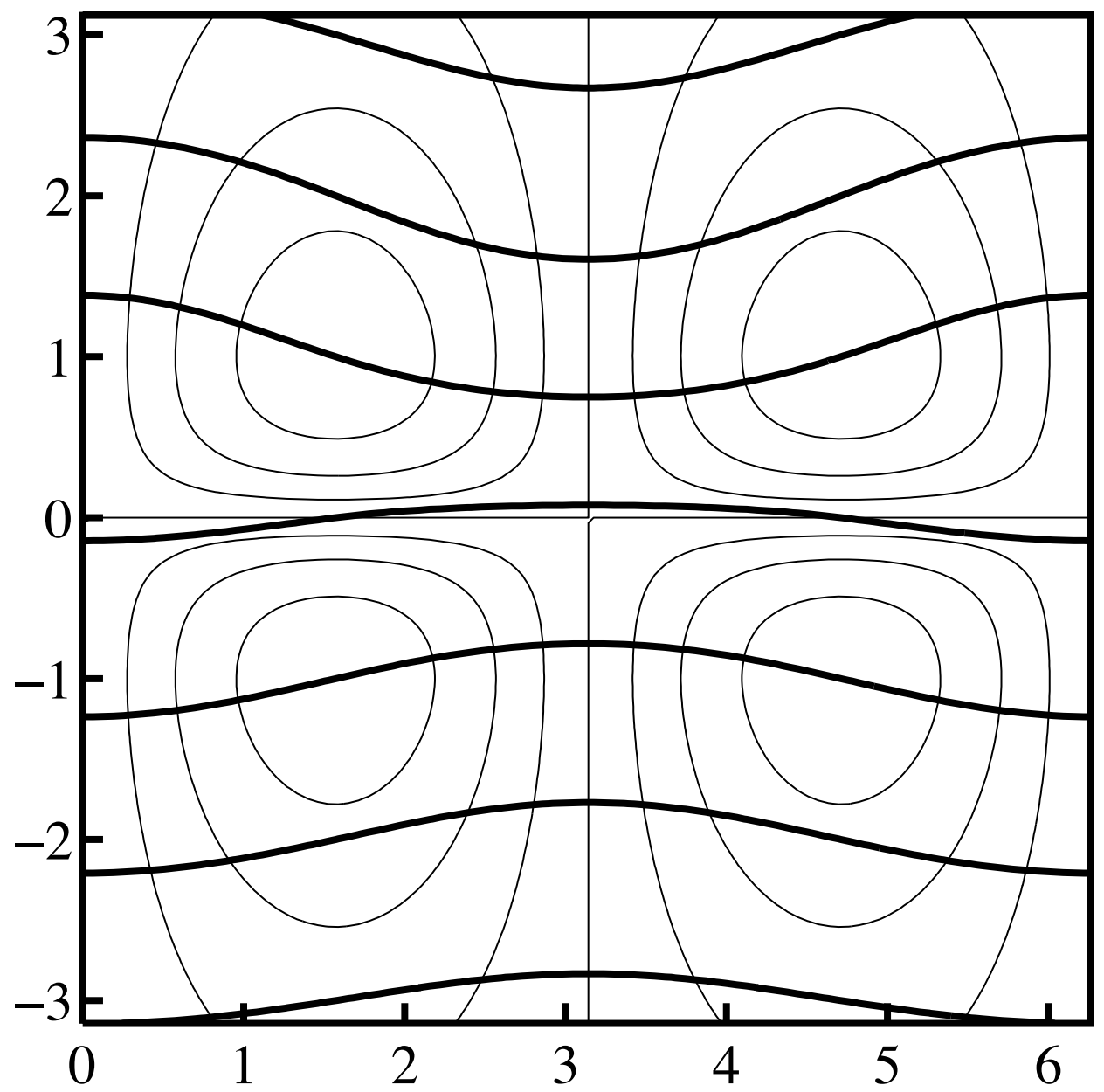

FiguRE 13. Streamfunction contours (light lines) and temperature contours for the large-wavenumber Marangoni instability for the inert case with $a=1, k^{*}=1.0$, and $\kappa^{*}=0.5$. The magnitude of the perturbation is exaggerated to emphasize the deformation of the temperature contours. 


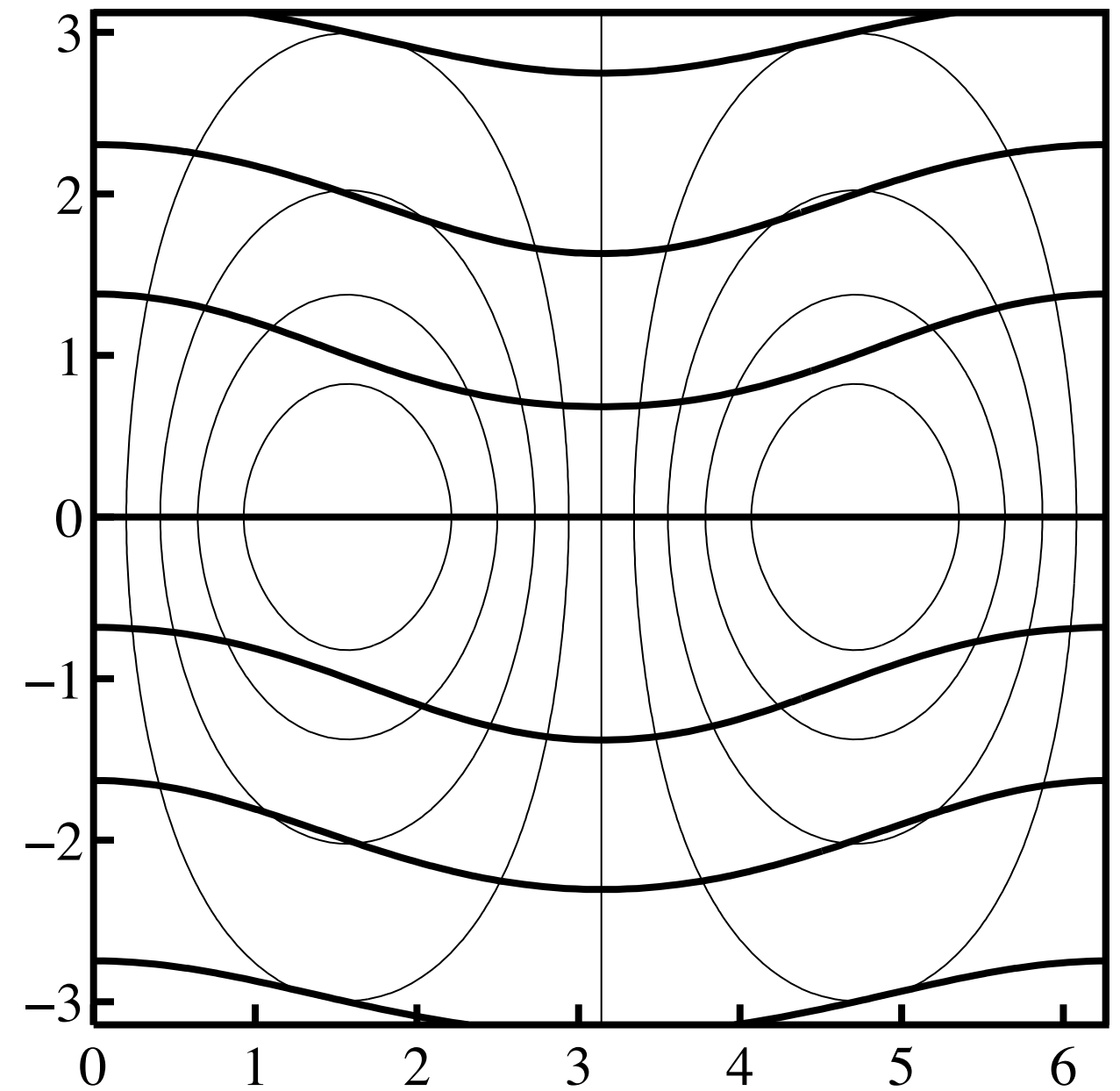

FiguRE 14. Streamfunction contours (light lines) and temperature contours for the large-wavenumber solution for the two-phase case with $a=1$ and equal material properties in both phases. The magnitude of the perturbation is exaggerated to emphasize the deformation of the temperature contours. 